\title{
REAL STRUCTURES AND THE Pin'(2)-MONOPOLE EQUATIONS
}

\author{
NOBUHIRO NAKAMURA
}

\begin{abstract}
We investigate the $\mathrm{Pin}^{-}(2)$-monopole invariants of symplectic 4-manifolds and Kähler surfaces with real structures. We prove a nonvanishing theorem for real symplectic 4-manifolds which is an analogue of Taubes' nonvanishing theorem of the Seiberg-Witten invariants for symplectic 4-manifolds. Furthermore, the Kobayashi-Hitchin type correspondence for real Kähler surfaces is given.
\end{abstract}

\section{Introduction}

In the study of the Seiberg-Witten invariants, the computations of the invariants of Kähler surfaces are fundamental [3, 6, 7, 16, 27], and they are based on a certain type of Kobayashi-Hitchin correspondence. On the other hand, Taubes' works [23 26] on the Seiberg-Witten theory on symplectic 4manifolds begin with the nontriviality theorem [23] for the canonical $\mathrm{Spin}^{c}$ structure.

The purpose of this paper is to prove the theorems for $\mathrm{Pin}^{-}(2)$-monopole invariants [18 parallel to the above results. The $\mathrm{Pin}^{-}(2)$-monopole equations [17, 18] are a variant of the Seiberg-Witten equations twisted along a local system associated with a double covering $X \rightarrow \hat{X}$. In fact, an anti-linear involution $I$ on a $\operatorname{Spin}^{c}$ structure $\mathfrak{s}$ on the double cover $X$ is defined, and the $\mathrm{Pin}^{-}(2)$-monopole theory on $\hat{X}$ can be identified with the $I$-invariant part of the Seiberg-Witten theory on $(X, \mathfrak{s})$. We study Kähler surfaces and symplectic 4-manifolds with real structure. Our starting point is to understand the aforementioned $I$-action through the real structure.

Let us state our results more precisely. Let $(X, \omega, \iota)$ be a closed real symplectic 4-manifold, where $X$ is a closed smooth 4 -manifold $X, \omega$ is a symplectic form and $\iota$ is an involution on $X$ such that $\iota^{*} \omega=-\omega$. Let $J$ be a compatible almost complex structure such that $\iota_{*} \circ J=-J \circ \iota_{*}$. Let $K$ be the canonical complex line bundle associated with $J$. We assume $(X, \omega, \iota)$ has empty real part, that is, the involution $\iota$ is free. Let $\hat{X}$ be the quotient manifold $X / \iota$ and $\pi: X \rightarrow \hat{X}$ the projection. Since $\iota$ induces an anti-linear involution on $K$, the quotient bundle $\hat{K}:=K / \iota$ is a nonorientable $\mathbb{R}^{2}$ bundle. Let $\ell=X \times_{\{ \pm 1\}} \mathbb{Z}$ be the local system associated to the double cover $X \rightarrow \hat{X}$. Let $\ell_{\mathbb{R}}:=\ell \otimes \mathbb{R}=\operatorname{det} \hat{K}$.

The $\mathrm{Pin}^{-}(2)$-monopole equations are defined on a $\mathrm{Spin}^{c_{-}}$structure, which is a $\mathrm{Pin}^{-}(2)$-analogue of $\mathrm{Spin}^{c}$ structure. In the above situation, we define the canonical Spin ${ }^{c_{-}}$structure $\hat{\mathfrak{s}}_{0}$ associated with the real structure (\$2.2). The following theorem is an analogue of Taubes' nonvanishing theorem [23].

2010 Mathematics Subject Classification. 57R57, 53C55, 53D05. 
Theorem 1.1. Suppose

(1) $w_{2}(\hat{X})+w_{2}(\hat{K})+w_{1}\left(\ell_{\mathbb{R}}\right)^{2}=0$,

(2) $\pi^{*}: H^{1}\left(\hat{X} ; \mathbb{Z}_{2}\right) \rightarrow H^{1}\left(X ; \mathbb{Z}_{2}\right)$ is surjective.

Then there exists a unique canonical Spin $^{c_{-}}$structure $\hat{\mathfrak{s}}_{0}$ on $X \rightarrow \hat{X}$. Furthermore, suppose $b_{+}^{\ell}=\operatorname{dim} H^{+}\left(\hat{X} ; \ell_{\mathbb{R}}\right) \geq 2$. Then the $\operatorname{Pin}^{-}(2)$-monopole invariant $\mathrm{SW}^{\operatorname{Pin}}\left(\hat{X}, \hat{\mathfrak{s}}_{0}\right)$ is \pm 1 .

Remark 1.2. We refer the readers to [17,18] for the generality of the $\mathrm{Pin}^{-}(2)-$ monopole theory. In general, the $\mathrm{Pin}^{-}(2)$-monopole invariant is a $\mathbb{Z}_{2}$-valued invariant. We can define its $\mathbb{Z}$-valued refinement in some cases, e.g., when the moduli space is 0 -dimensional and orientable. The statement that $\mathrm{SW}^{\mathrm{Pin}}\left(\hat{X}, \hat{\mathfrak{s}}_{0}\right)= \pm 1$ means that the invariant is nonzero anyway, and if its $\mathbb{Z}$-valued refinement is defined, then its value is \pm 1 .

Like the ordinary Seiberg-Witten theory, the Pin ${ }^{-}(2)$-monopole theory has a symmetry of conjugation (\$2.3). The conjugate of the canonical Spin ${ }^{c_{-}}$ structure $\hat{\mathfrak{s}}_{0}$ is the anti-canonical Spin $^{c_{-}}$structure $\hat{\mathfrak{s}}_{0} \hat{\otimes} \hat{K}$ which is obtained by twisting $\hat{\mathfrak{s}}_{0}$ by $\hat{K}$. Theorem 1.1 with Corollary 2.14 implies the following.

Corollary 1.3. $\mathrm{SW}^{\mathrm{Pin}}\left(\hat{X}, \hat{\mathfrak{s}}_{0} \hat{\otimes} \hat{K}\right)= \pm 1$.

An $\mathrm{O}(2)$ bundle $\hat{L}$ called characteristic bundle is associated with a $\mathrm{Spin}^{c_{-}}$ structure. Let $\tilde{c}_{1}(\hat{L}) \in H^{2}(\hat{X} ; \ell)$ be its $\ell$-coefficient Euler class. Since $\iota^{*} \omega=$ $-\omega$, there is a $\ell_{\mathbb{R}}$-valued self-dual closed 2 -form $\hat{\omega} \in \Omega^{2}\left(X ; \ell_{\mathbb{R}}\right)$ such that $\pi^{*} \hat{\omega}=\omega$. The following is an analogue of [24, Theorem 2].

Theorem 1.4. Under the assumptions of Theorem 1.1, if the $\mathrm{Pin}^{-}(2)-$ monopole invariant for a $\mathrm{Spin}^{c_{-}}$structure $\hat{\mathfrak{s}}$ on $X \rightarrow \hat{X}$ is nonzero, then its characteristic bundle $\hat{L}$ satisfies

$$
\left|\tilde{c}_{1}(\hat{L}) \cdot[\hat{\omega}]\right| \leq \tilde{c}_{1}(\hat{K}) \cdot[\hat{\omega}]
$$

and the virtual dimension $d(\hat{\mathfrak{s}})$ of the moduli space is 0 .

Suppose further that $(X, \omega)$ is a compact Kähler surface and $\iota$ is an antiholomorphic free involution. In such a case, we prove a certain kind of Kobayashi-Hitchin correspondence (\$4). In fact, the $\mathrm{Pin}^{-}(2)$-monopole moduli space for $\hat{X}$ can be identified with the $I$-invariant part of the space of simple holomorphic pairs of holomorphic structures on a line bundle with nonzero holomorphic sections. (Furthermore, a simple holomorphic pair is identified with an effective divisor on $X$.) By using this description, we can compute the $\mathrm{Pin}^{-}(2)$-monopole invariants for the quotient manifolds of several kinds of Kähler surfaces. The following is an analogue of [16, Theorem 7.4.1].

Theorem 1.6. Let $X$ be a minimal Kähler surface of general type. Suppose $\iota: X \rightarrow X$ is an anti-holomorphic involution without fixed points satisfying the assumptions in Theorem 1.1. Then

$$
\mathrm{SW}^{\operatorname{Pin}}(\hat{X}, \hat{\mathfrak{s}})=\left\{\begin{array}{cc} 
\pm 1 & \hat{\mathfrak{s}}=\hat{\mathfrak{s}}_{0} \text { or } \hat{\mathfrak{s}}_{0} \hat{\otimes} \hat{K} \\
0 & \text { otherwise }
\end{array}\right.
$$


The hypersurfaces in $\mathbb{C P}^{3}$ with complex conjugation satisfy the conditions of Theorem 1.6 (\$5.1). We also compute the invariants of elliptic surfaces in $\$ 5.2$.

Acknowledgements. The author would like to thank the referee for his comments on the earlier version of the paper. The author is supported in part by JSPS Grant-in-Aid for Scientific Research (C) 25400096 and 19K03506.

\section{Spin $^{c_{-}}$structures induced from the real structure}

2.1. Reduction of the frame bundle. Recall the isomorphism $\mathrm{U}(2) \cong$ $(\mathrm{U}(1) \times \mathrm{SU}(2)) /\{ \pm 1\}$. Define the group $\hat{\mathrm{U}}(2)$ by

$$
\hat{\mathrm{U}}(2)=\left(\mathrm{Pin}^{-}(2) \times \mathrm{SU}(2)\right) /\{ \pm 1\} \text {. }
$$

Then $\hat{\mathrm{U}}(2) / \mathrm{Pin}^{-}(2)=\mathrm{SO}(3), \hat{\mathrm{U}}(2) / \mathrm{SU}(2)=\mathrm{O}(2)$, the identity component of $\hat{U}(2)$ is $U(2)$, and $\hat{U}(2) / U(2)=\{ \pm 1\}$. We have an exact sequence

$$
1 \rightarrow\{ \pm 1\} \rightarrow \hat{\mathrm{U}}(2) \stackrel{\sigma}{\rightarrow} \mathrm{O}(2) \times \mathrm{SO}(3) \rightarrow 1 .
$$

Note that $\hat{\mathrm{U}}(2)$ is embedded in $\mathrm{SO}(4)$ as

$$
\hat{\mathrm{U}}(2)=\frac{\mathrm{Pin}^{-}(2) \times \mathrm{SU}(2)}{\{ \pm 1\}} \subset \frac{\mathrm{SU}(2) \times \mathrm{SU}(2)}{\{ \pm 1\}}=\mathrm{SO}(4) .
$$

Suppose we have a manifold $\hat{Y}$ with a double covering $Y \rightarrow \hat{Y}$ and a principal $\hat{\mathrm{U}}(2)$-bundle $P$ over $\hat{Y}$ such that $P / \mathrm{U}(2) \cong Y$. Then we have an $\mathrm{O}(2)$ bundle $P_{O}:=P / \mathrm{SU}(2)$ such that $P_{O} / \mathrm{SO}(2) \cong Y$ and an $\mathrm{SO}(3)$-bundle $P_{S}:=P / \operatorname{Pin}^{-}(2)$. Conversely, the following holds

Proposition 2.3. For a double covering $Y \rightarrow \hat{Y}$, let $\ell_{\mathbb{R}}=Y \times_{\{ \pm 1\}} \mathbb{R}$ and suppose an $\mathrm{O}(2)$-bundle $P_{O}$ such that $P_{O} / \mathrm{SO}(2) \cong Y$ and an $\mathrm{SO}(3)$-bundle $P_{S}$ are given. If $w_{2}\left(P_{O}\right)+w_{1}\left(\ell_{\mathbb{R}}\right)^{2}=w_{2}\left(P_{S}\right)$, then there exists a $\hat{\mathrm{U}}(2)$-bundle $P$ such that

$$
P / \mathrm{Pin}^{-}(2) \cong P_{S}, \quad P / \mathrm{SU}(2) \cong P_{O}, \quad P / \mathrm{U}(2) \cong Y .
$$

Proof. (Cf. [17, Proposition 11].) Note that the image of $\mathrm{Pin}^{-}(2) \subset \mathrm{Sp}(1)=$ $\operatorname{Spin}(3)$ by the canonical homomorphism $\mathrm{Spin}(3) \rightarrow \mathrm{SO}(3)$ is a copy of $\mathrm{O}(2)$ embedded in $\mathrm{SO}(3)$. The embedding $\mathrm{O}(2) \subset \mathrm{SO}(3)$ is given by $A \mapsto$ $A \oplus \operatorname{det} A$. Embed $\mathrm{O}(2) \times \mathrm{SO}(3)$ into $\mathrm{SO}(6)$ by using this embedding. Then we have a commutative diagram

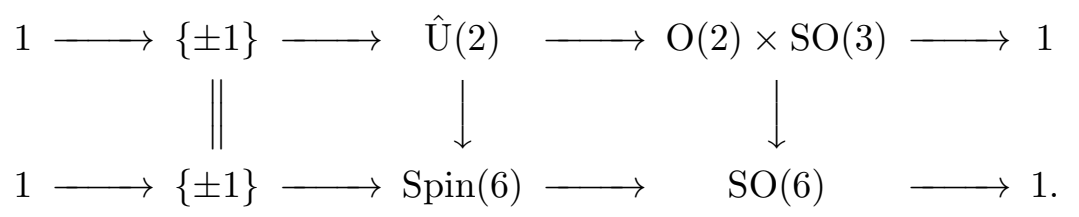

The diagram leads to a commutative diagram of fibrations

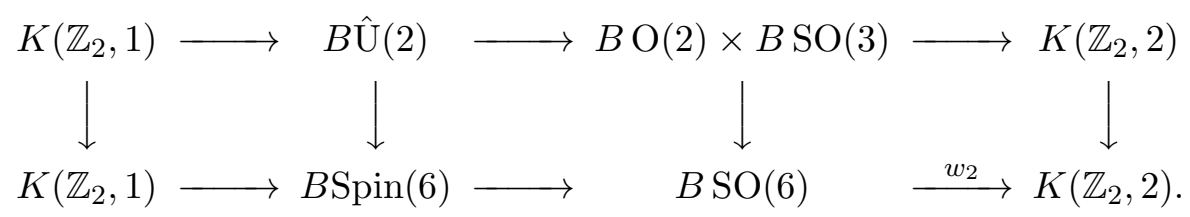


From these, we see that

$$
w_{2}\left(P_{S} \oplus P_{O} \oplus \operatorname{det} P_{O}\right)=w_{2}\left(P_{S}\right)+w_{2}\left(P_{O}\right)+w_{1}\left(\ell_{\mathbb{R}}\right)^{2}=0
$$

is the required condition.

Remark 2.4. The choice of $P$ is not unique. The possibility of $P$ is parametrized by $H^{1}\left(\hat{Y} ; \mathbb{Z}_{2}\right)$.

Recall the embedding

$$
\mathrm{U}(2)=(\mathrm{U}(1) \times \mathrm{SU}(2)) /\{ \pm 1\} \subset(\mathrm{SU}(2) \times \mathrm{SU}(2)) /\{ \pm 1\}=\mathrm{SO}(4)
$$

and a commutative diagram

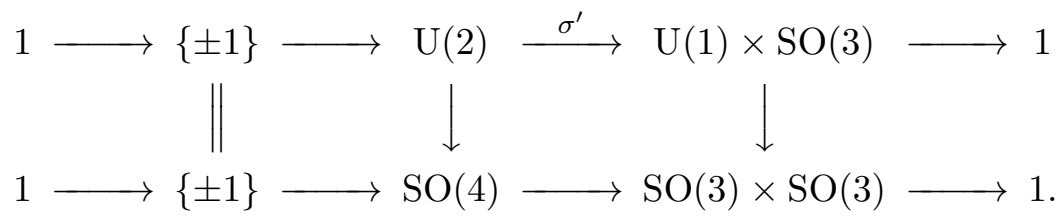

Let $(X, \omega, J)$ be a symplectic 4-manifold with compatible almost complex structure $J$. Fixing a Hermitian metric on $T X$, we obtain a $\mathrm{U}(2)$ reduction $P_{F}$ of the $\mathrm{SO}(4)$-frame bundle. Then we have a $\mathrm{U}(1)$-bundle $P_{K}:=$ $P_{F} / \mathrm{SU}(2)$ and an $\mathrm{SO}(3)$-bundle $P_{S}:=P_{F} / \mathrm{U}(1)$. Let $K=\Lambda^{2,0}(X)$ and $K^{-1}=\Lambda^{0,2}(X)$ be respectively the canonical and anti-canonical line bundles associated with the almost complex structure $J$. Note that $\Lambda^{+}(X) \otimes_{\mathbb{R}} \mathbb{C} \cong$ $\mathbb{C} \omega \oplus K \oplus K^{-1}$. Then we can identify

$$
P_{K} \times_{\mathrm{U}(1)} \mathbb{C} \cong K \cong K^{-1}
$$

as real vector bundles. We assume $P_{K} \times_{\mathrm{U}(1)} \mathbb{C}=K$. On the other hand,

$$
\Lambda^{-}(X) \cong P_{S} \times_{\mathrm{SO}(3)} \mathbb{R}^{3} .
$$

Let $(X, \omega, \iota)$ be a closed real symplectic 4-manifold without real part. Then $X$ admits an almost complex structure $J$ compatible to $\omega$ such that $\iota_{*} \circ J=-J \circ \iota_{*}$. Fixing such a $J$, we have a $\mathrm{U}(2)$ reduction $P_{F}$ of the $\mathrm{SO}(4)$ frame bundle. Let $P_{K}$ and $P_{S}$ be the induced $\mathrm{U}(1)$ and $\mathrm{SO}(3)$ bundles. Let $\hat{X}$ be the quotient manifold $\hat{X}=X / \iota$ and $\ell_{\mathbb{R}}=X \times_{\{ \pm 1\}} \mathbb{R}$. The involution $\iota$ induces a bundle automorphism $\tilde{\iota}$ of $P_{S}$ such that $\tilde{\iota}^{2}=1$, and its quotient bundle $\hat{P}_{S}=P_{S} / \tilde{\iota}$ over $\hat{X}$ has the property that

$$
\hat{P}_{S} \times \mathrm{SO}(3) \mathbb{R}^{3}=\Lambda^{-}(\hat{X}) .
$$

On the other hand, $\iota$ does not induce a bundle automorphism on $P_{K}$ since $\iota$ is not complex linear. However $\iota$ induces an anti-linear involution on the canonical bundle $K=P_{K} \times_{\mathrm{U}(1)} \mathbb{C}$. Then the quotient bundle $\hat{K}=K / \iota$ is a nonorientable $\mathbb{R}^{2}$ bundle over $\hat{X}$ such that $\operatorname{det} \hat{K}=\ell_{\mathbb{R}}$. Let $\hat{P}_{K}$ be the $\mathrm{O}(2)$-bundle over $\hat{X}$ of orthogonal frames on $\hat{K}$. By Proposition 2.3, we have a $\hat{\mathrm{U}}(2)$-bundle $\hat{P}$ which induces $\hat{P}_{S}$ and $\hat{P}_{K}$ if $w_{2}(\hat{X})=w_{2}(\hat{K})+w_{1}\left(\ell_{\mathbb{R}}\right)^{2}$. Note that the $\{ \pm 1\}$-bundle $\hat{P} / \mathrm{U}(2) \rightarrow \hat{X}$ is isomorphic to $\pi: X \rightarrow \hat{X}$. Fix an isomorphism between them. Then $\hat{P} \rightarrow \hat{P} / \mathrm{U}(2)$ can be considered as a $\mathrm{U}(2)$-bundle over $X$. This $\mathrm{U}(2)$-bundle $\hat{P} \rightarrow \hat{P} / \mathrm{U}(2)=X$ is denoted by $P^{\prime}$.

Proposition 2.5. Suppose

(1) $w_{2}(\hat{X})+w_{2}(\hat{K})+w_{1}\left(\ell_{\mathbb{R}}\right)^{2}=0$, 
(2) $\pi^{*}: H^{1}\left(\hat{X} ; \mathbb{Z}_{2}\right) \rightarrow H^{1}\left(X ; \mathbb{Z}_{2}\right)$ is surjective.

Then we can take $a \hat{\mathrm{U}}(2)$-bundle $\hat{P} \rightarrow \hat{X}$ such that

$$
\hat{P} / \operatorname{Pin}^{-}(2) \cong \hat{P}_{S}, \quad \hat{P} / \mathrm{SU}(2) \cong \hat{P}_{K}, \quad \hat{P} / \mathrm{U}(2) \cong X, \quad P^{\prime} \cong P_{F} .
$$

Furthermore $\hat{T}=\hat{P} \times_{\hat{\mathrm{U}}(2)} \mathbb{R}^{4}$ is isomorphic to $T \hat{X}$, where $\hat{T}$ is defined via the embedding (2.2).

Proof. By the proof of Proposition 2.3, we see that the set of isomorphism classes of $\hat{\mathrm{U}}(2)$-bundle $\hat{P}$ which induces the same $\hat{P}_{K}$ and $\hat{P}_{S}$ is parametrized by $H^{1}\left(\hat{X} ; \mathbb{Z}_{2}\right)$. If a choice of $\hat{P}$ is given, then every other choice is obtained by tensoring a real line bundle. Similarly, the set of isomorphism classes of U(2)-bundle $P_{F}$ which induces the same $P_{K}$ and $P_{S}$ is parametrized by $H^{1}\left(X ; \mathbb{Z}_{2}\right)$. Now choose $\hat{P}$ which induces $\hat{P}_{K}$ and $\hat{P}_{S}$. Then it follows from the construction that the $\mathrm{U}(2)$-bundle $P^{\prime}$ induces $P_{K}$ and $P_{S}$. Thus the difference between $P_{F}$ and $P^{\prime}$ is given by an element of $H^{1}\left(X ; \mathbb{Z}_{2}\right)$. Under the assumption, the difference can be annihilated by tensoring an appropriate real line bundle over $\hat{X}$ with $\hat{P}$.

Since $\pi^{*} \hat{P} \times_{\hat{\mathrm{U}}(2)} \mathbb{R}^{4}=P^{\prime} \times_{\mathrm{U}(2)} \mathbb{R}^{4}=P_{F} \times_{\mathrm{U}(2)} \mathbb{R}^{4}=T X$, we have $\pi^{*} \hat{T} \cong T X \cong \pi^{*} T \hat{X}$. From this, it follows that $e(\hat{T})=e(T \hat{X})$ and $p_{1}(\hat{T})=$ $p_{1}(T \hat{X})$. Consider the homomorphisms $\hat{\mathrm{U}}(2) \stackrel{\sigma}{\rightarrow} \mathrm{O}(2) \times \mathrm{SO}(3) \stackrel{p}{\rightarrow} \mathrm{SO}(3)$ where $p$ is the projection to the second factor. Then the composite map $p \circ \sigma: \hat{\mathrm{U}}(2) \rightarrow \mathrm{SO}(3)$ factors through $\hat{\mathrm{U}}(2) \hookrightarrow \mathrm{SO}(4) \rightarrow \mathrm{SO}(3)$. Then we have a commutative diagram

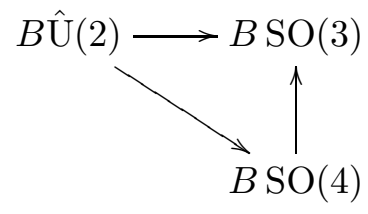

From this, it follows that $w_{2}(\hat{T})=w_{2}\left(\hat{P}_{S}\right)=w_{2}(\hat{X})$. Therefore $\hat{T} \cong T \hat{X}$.

Remark 2.7. The choice of $\hat{P}$ is not unique. The possibility of $\hat{P}$ is parametrized by $\operatorname{ker}\left(\pi^{*}: H^{1}\left(\hat{X} ; \mathbb{Z}_{2}\right) \rightarrow H^{1}\left(X ; \mathbb{Z}_{2}\right)\right)$.

2.2. Canonical Spin ${ }^{c_{-}}$structure. Recall that the canonical $\operatorname{Spin}^{c}$ structure $\mathfrak{s}_{0}$ over $X$ with respect to the almost complex structure $J$ is defined from the $\mathrm{U}(2)$-reduction $P_{F}$, and it has the positive spinor bundle $W_{0}^{+}$of the form $W_{0}^{+}=\underline{\mathbb{C}} \oplus K^{-1}$. In this subsection, we define the canonical Spin ${ }^{c_{-}}$ structure over $X \rightarrow \hat{X}$ induced from the real structure on $X$.

Recall that

$$
\operatorname{Spin}^{c_{-}}(4)=\frac{\mathrm{SU}(2) \times \mathrm{SU}(2) \times \operatorname{Pin}^{-}(2)}{\{ \pm 1\}}=\frac{\operatorname{Sp}(1) \times \operatorname{Sp}(1) \times \operatorname{Pin}^{-}(2)}{\{ \pm 1\}} .
$$

A Spin ${ }^{c_{-}}$structure $\hat{\mathfrak{s}}$ on $X \rightarrow \hat{X}$ consists of a $\operatorname{Spin}^{c_{-}}(4)$-bundle $Q$ over $\hat{X}$, an isomorphism of $\mathbb{Z} / 2$-bundles $Q / \operatorname{Spin}^{c}(4) \cong X$, and an isomorphism between the $\mathrm{SO}(4)$-frame bundle and $Q / \mathrm{Pin}^{-}(2)$. The $\mathrm{O}(2)$-bundle $\hat{L}=Q / \operatorname{Spin}(4)$ is called the characteristic bundle of $\hat{\mathfrak{s}}$. The bundle $\hat{L}$ has a $\ell$-coefficient orientation and its Euler class is denoted by $\tilde{c}_{1}(\hat{L}) \in H^{2}(\hat{X} ; \ell)$. We often 
make no distinction between $\hat{L}$ and its associated $\mathbb{R}^{2}$-bundle. Let $\mathbb{H}_{ \pm}$be the Spin $^{c_{-}}(4)$ modules which are copies of $\mathbb{H}$ as vector spaces such that the action of $\left[q_{+}, q_{-}, u\right] \in \operatorname{Spin}^{c_{-}}(4)=\left(\operatorname{Sp}(1) \times \operatorname{Sp}(1) \times \operatorname{Pin}^{-}(2)\right) /\{ \pm 1\}$ on $\phi \in \mathbb{H}_{ \pm}$is given by $q_{ \pm} \phi u^{-1}$. Then the associated bundles $W_{ \pm}=Q \times \times_{\operatorname{Spin}^{c-}(4)} \mathbb{H}_{ \pm}$are the spinor bundles of $\hat{\mathfrak{s}}$.

Note that the embedding $\hat{\mathrm{U}}(2) \hookrightarrow \mathrm{SO}(4)$ factors through another embed$\operatorname{ding} \varepsilon: \hat{\mathrm{U}}(2) \rightarrow \operatorname{Spin}^{c_{-}}(4)$ which is defined by

$$
\begin{aligned}
\varepsilon: \hat{\mathrm{U}}(2)=\frac{\mathrm{Pin}^{-}(2) \times \mathrm{SU}(2)}{\{ \pm 1\}} & \rightarrow \frac{\mathrm{SU}(2) \times \mathrm{SU}(2) \times \mathrm{Pin}^{-}(2)}{\{ \pm 1\}}=\operatorname{Spin}^{c_{-}}(4), \\
(u, q) & \mapsto(u, q, u) .
\end{aligned}
$$

When we have a $\hat{\mathrm{U}}(2)$-bundle $\hat{P}$ as in Proposition 2.5, a $\operatorname{Spin}^{c_{-}}$structure $\hat{\mathfrak{s}}$ over $X$ is defined via the embedding $\varepsilon$. The $\operatorname{Spin}^{c_{-}}(4)$-bundle $Q$ of $\hat{\mathfrak{s}}$ is given by

$$
Q=\hat{P} \times_{\hat{\mathrm{U}}(2)} \operatorname{Spin}^{c_{-}}(4),
$$

Note that the characteristic $\mathrm{O}(2)$-bundle of $\hat{\mathfrak{s}}$ is $\hat{P}_{K}$. The positive spinor bundle $\hat{W}^{+}$is defined by the adjoint action of $\operatorname{Pin}^{-}(2)$ on the space of quaternions $\mathbb{H}=\mathbb{C} \oplus j \mathbb{C}$ :

$$
W^{+}=Q \times_{\operatorname{Spin}^{c-}(4)} \mathbb{H}_{+}=\hat{P} \times_{\operatorname{Pin}^{-}(2)} \mathbb{H} .
$$

For $u \in \mathrm{U}(1)$ and $z \in \mathbb{C}$, the adjoint action is given by

$$
\begin{aligned}
\operatorname{ad}_{u}(z) & =u z u^{-1}=z, \\
\operatorname{ad}_{j u}(z) & =j u z u^{-1} j^{-1}=\bar{z}, \\
\operatorname{ad}_{u}(j z) & =u^{2} j z=u^{2} \bar{z} j, \\
\operatorname{ad}_{j u}(j z) & =u^{-2} j \bar{z}=u^{-2} z j .
\end{aligned}
$$

This action preserves the components $\mathbb{C}$ and $j \mathbb{C}$. It follows from (2.8) that $\hat{W}^{+}$is decomposed into the direct sum of two $\mathbb{R}^{2}$ bundles as $\hat{W}^{+}=\hat{E}_{1} \oplus \hat{E}_{2}$ such that $\operatorname{det} \hat{E}_{1}=\operatorname{det} \hat{E}_{2}=\ell_{\mathbb{R}}$. Define the $\mathbb{R}^{2}$-bundle $\underline{\hat{\mathbb{C}}}$ by $\underline{\mathbb{C}}=X \times_{\{ \pm 1\}} \mathbb{C}$, where $\{ \pm 1\}$ acts on $\mathbb{C}$ by complex conjugation. Note that $\underline{\hat{\mathbb{C}}}=\underline{\mathbb{R}} \oplus \ell_{\mathbb{R}}$. Since $\pi^{*} \hat{W}^{+}=W_{0}^{+}=\underline{\mathbb{C}} \oplus K^{-1}$, we see that $\hat{W}^{+}$has a form of

$$
\hat{W}^{+}=\left(\underline{\hat{\mathbb{C}}} \oplus \hat{K}^{-1}\right) \otimes \lambda^{\prime},
$$

where $\hat{K}^{-1}=\left(K^{-1}\right) / \iota$ (which is the characteristic bundle of the $\mathrm{Spin}^{c_{-}}$ structure) and $\lambda^{\prime}$ is a real line bundle over $\hat{X}$ with $\pi^{*} \lambda^{\prime}$ trivial. Note that tensoring $\lambda^{\prime}$ to $\hat{P}$ changes $\hat{W}^{+}$into $\hat{W}_{0}^{+}=\underline{\mathbb{C}} \oplus \hat{K}^{-1}$. Now we define the canonical Spin ${ }^{c_{-}}$structure.

Definition 2.9. A Spin ${ }^{c_{-}}$structure $\mathfrak{s}_{0}$ on $X \rightarrow \hat{X}$ is canonical if it is defined from a $\hat{\mathrm{U}}(2)$-bundle $\hat{P}$ satisfying (2.6) and its positive spinor bundle $\hat{W}_{0}^{+}$has a form of

$$
\hat{W}_{0}^{+}=\underline{\hat{\mathbb{C}}} \oplus \hat{K}^{-1} .
$$

The above discussion implies the following.

Corollary 2.10. Suppose (1) and (2) in Theorem 1.1. Then there exists a unique canonical $\mathrm{Spin}^{c_{-}}$structure on $X \rightarrow \hat{X}$. 
Recall $\mathbb{R}^{2}$-bundles $\hat{E}$ such that $\operatorname{det} \hat{E}=\ell_{\mathbb{R}}$ with $\ell_{\mathbb{R}^{-}}$-coefficient orientation are classified by $\tilde{c}_{1}(\hat{E}) \in H^{2}(\hat{X} ; \ell)$. We call an $\mathbb{R}^{2}$-bundle $\hat{E}$ such that $\operatorname{det} \hat{E}=\ell_{\mathbb{R}}$ an $\mathbb{R}^{2}$-bundle twisted along $\ell_{\mathbb{R}}$. For $\mathbb{R}^{2}$-bundles $\hat{E}_{1}$ and $\hat{E}_{2}$ twisted along $\ell_{\mathbb{R}}$, there exists another $\mathbb{R}^{2}$-bundle $\hat{E}$ twisted along $\ell_{\mathbb{R}}$ such that $\tilde{c}_{1}(\hat{E})=\tilde{c}_{1}\left(\hat{E}_{1}\right)+\tilde{c}_{1}\left(\hat{E}_{2}\right)$, which can be considered as a "twisted tensor product" of $\hat{E}_{1}$ and $\hat{E}_{2}$. We write $\hat{E}=\hat{E}_{1} \hat{\otimes} \hat{E}_{2}$.

If $X \rightarrow \hat{X}$ admits a Spin ${ }^{c_{-}}$structure, then the set of equivalence classes of Spin $^{c_{-}}$structures is also parametrized by $H^{2}(X ; \ell)$. Once a Spin ${ }^{c_{-}}$structure is given, the other $\operatorname{Spin}^{c_{-}}$structures are given by "tensoring" an $\mathbb{R}^{2}$-bundle $\hat{E}$ twisted along $\ell_{\mathbb{R}}$. In fact, when we have a canonical $\operatorname{Spin}^{c_{-}}$structure $\hat{\mathfrak{s}}_{0}$, there is a Spin $^{c_{-}}$structure whose positive spinor bundle is

$$
\hat{W}=\hat{E} \oplus\left(\hat{E} \hat{\otimes} \hat{K}^{-1}\right) .
$$

This $\operatorname{Spin}^{c_{-}}$structure is denoted by $\hat{\mathfrak{s}}_{0} \hat{\otimes} \hat{E}$.

Definition 2.11. The Spin ${ }^{c_{-}}$structure $\hat{\mathfrak{s}}_{0} \hat{\otimes} \hat{K}$ is called the anti-canonical Spin $^{c_{-}}$structure. This has the spinor bundle of the form

$$
\hat{W}=\hat{K} \oplus \underline{\hat{\mathbb{C}}} .
$$

Remark 2.12. For a $\operatorname{Spin}^{c_{-}}$structure $\hat{\mathfrak{s}}$ over $\pi: X \rightarrow \hat{X}$, let $\pi^{*} \hat{\mathfrak{s}}$ be the $\operatorname{Spin}^{c}$ structure over $X$ which is the pull-back of $\hat{\mathfrak{s}}$. Then $\pi^{*} \hat{\mathfrak{s}}$ has two $\operatorname{Spin}^{c}$ reductions, and one of them is the canonical reduction [18, §2.4]. Then it can be seen that the canonical reduction of the pull-back $\pi^{*} \hat{\mathfrak{s}}_{0}$ of the canonical Spin ${ }^{c_{-}}$structure $\hat{\mathfrak{s}}_{0}$ is the canonical $\operatorname{Spin}^{c}$ structure $\mathfrak{s}_{0}$ on $X$, and the canonical reduction of $\pi^{*}\left(\hat{\mathfrak{s}}_{0} \hat{\otimes} \hat{K}\right)$ is the anti-canonical Spin ${ }^{c}$ structure $\mathfrak{s}_{0} \otimes K$.

2.3. A symmetry in the $\operatorname{Pin}^{-}(2)$-monopole theory. It is well-known that there is a symmetry of complex conjugation in the Seiberg-Witten theory [16, §6.8]. We explain a similar symmetry in the $\mathrm{Pin}^{-}(2)$-monopole theory. The conjugation of a quaternion $z \in \mathbb{H}$ is given by

$$
z=a+i b+j c+k d \mapsto \bar{z}=a-i b-j c-k d .
$$

Define the conjugation $\alpha: \operatorname{Spin}^{c_{-}}(4) \rightarrow \operatorname{Spin}^{c_{-}}(4)$ by

$$
\alpha([q, z])=[q, \bar{z}] \text { for }[q, z] \in \operatorname{Spin}^{c_{-}}(4)=\operatorname{Spin}(4) \times_{\{ \pm 1\}} \operatorname{Pin}^{-}(2) .
$$

For a $\operatorname{Spin}^{c_{-}}$(4)-bundle $P$, let $P^{c}$ be the $\operatorname{Spin}^{c_{-}}(4)$-bundle such that the total space is same with $P$, but the action of $\operatorname{Spin}^{c_{-}}(4)$ is given by $p \cdot \alpha(q)$ for $p \in P=P^{c}$ and $q \in \operatorname{Spin}^{c_{-}}(4)$.

For a $\operatorname{Spin}^{c_{-}}$structure $\hat{\mathfrak{s}}$ with $\operatorname{Spin}^{c_{-}}(4)$-bundle $P$, we have a Spin ${ }^{c_{-}}$structure $\hat{\mathfrak{s}}^{c}$ whose $\operatorname{Spin}^{c-}(4)$-bundle is $P^{c}$. We call $\hat{\mathfrak{s}}^{c}$ the conjugate of $\hat{\mathfrak{s}}$.

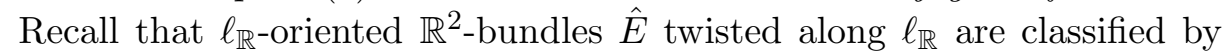
$\tilde{c}_{1}(\hat{E})$. For such an $\hat{E}$, let $\hat{E}^{c}$ be an $\mathbb{R}^{2}$-bundle such that $\tilde{c}_{1}\left(\hat{E}^{c}\right)=-\tilde{c}_{1}(\hat{E})$. We collect several facts on conjugate which can be easily seen.

Proposition 2.13. For a $\operatorname{Spin}^{c-}$ structure $\hat{\mathfrak{s}}$ and its conjugate $\hat{\mathfrak{s}}^{c}$, we have the following:

(1) If $\hat{L}$ is the characteristic bundle for $\hat{\mathfrak{s}}$, then $\hat{L}^{c}$ can be identified with the characteristic bundle of $\hat{\mathfrak{s}}^{c}$. In particular, $\tilde{c}_{1}\left(\hat{L}^{c}\right)=-\tilde{c}_{1}(\hat{L})$. 
(2) For an $\mathbb{R}^{2}$-bundle $E$ twisted along $\ell_{\mathbb{R}}$,

$$
(\hat{\mathfrak{s}} \hat{\otimes} \hat{E})^{c}=\hat{\mathfrak{s}}^{c} \hat{\otimes} \hat{E}^{c} .
$$

(3) The conjugate of the canonical $\mathrm{Spin}^{c_{-}}$structure is the anti-canonical Spin $^{c_{-}}$structure, i.e, $\hat{\mathfrak{s}}_{0}^{c}=\hat{\mathfrak{s}}_{0} \hat{\otimes} \hat{K}$.

(4) If $\mathfrak{s}$ is the canonical reduction of $\pi^{*} \hat{\mathfrak{s}}$, then the canonical reduction of $\pi^{*} \hat{\mathfrak{s}}^{c}$ is the complex conjugate $\overline{\mathfrak{s}}$ of $\mathfrak{s}$.

Let $\hat{\mathfrak{s}}$ be a $\operatorname{Spin}^{c_{-}}$structure on $\pi: X \rightarrow \hat{X}$ and $\mathfrak{s}$ the canonical reduction of $\pi^{*} \hat{\mathfrak{s}}$. By [17, $\left.\S 4.5\right]$ (see also [18, $\left.\S 2.5\right]$ ), there is an involution $I$ on the Seiberg-Witten theory on $(X, \mathfrak{s})$, and a bijective correspondence between the $\operatorname{Pin}^{-}(2)$-monopole solutions on $(\hat{X}, \hat{\mathfrak{s}})$ and the $I$-invariant Seiberg-Witten solutions on $(X, \mathfrak{s})$. Let us recall the relation between the downstairs $(\hat{X}, \hat{\mathfrak{s}})$ and upstairs $(X, \mathfrak{s})$ more precisely. Note that $\iota^{*} \mathfrak{s}$ is isomorphic to the complex conjugation $\overline{\mathfrak{s}}$ of $\mathfrak{s}$. For a configuration $(A, \phi)$ on $(X, \mathfrak{s}), I(A, \phi)$ is defined by

$$
I(A, \phi)=\left(\overline{\iota^{*} A}, \overline{\iota^{*} \phi}\right),
$$

where ${ }^{-}$means complex conjugation. For a configuration $(\hat{A}, \hat{\phi})$ on $(\hat{X}, \hat{\mathfrak{s}})$, we have a unique configuration $(A, \phi)$ on $(X, \mathfrak{s})$ such that $\phi=\pi^{*} \hat{\phi}$ and $A$ is the canonical $\mathrm{U}(1)$ reduction of the $\mathrm{O}(2)$-connection $\pi^{*} A$ which is the pull-back of $A$. We call $(A, \phi)$ the lift of $(\hat{A}, \hat{\phi})$. Note that the lift $(A, \phi)$ is $I$-invariant. by

The gauge transformation group of the $\mathrm{Pin}^{-}(2)$-monopole theory is given

$$
\hat{\mathcal{G}}=\Gamma\left(X \times_{\{ \pm 1\}} \mathrm{U}(1)\right),
$$

where $\{ \pm 1\}$ acts on $\mathrm{U}(1)$ by $u \mapsto u^{-1}$. Then $\hat{\mathcal{G}}$ can be identified with the $I$-invariant gauge transformation group on the upstairs $X$. That is, the $I$ action on $\mathcal{G}=C^{\infty}(X, \mathrm{U}(1))$ is given by $f \mapsto \overline{\iota^{*} f}$, and we have a natural identification $\hat{\mathcal{G}}=\mathcal{G}^{I}$.

The $\mathrm{Pin}^{-}(2)$-monopole moduli space is

$$
\hat{\mathcal{M}}(\hat{X}, \hat{\mathfrak{s}})=\left\{\operatorname{Pin}^{-}(2) \text {-monopole solutions on } \hat{\mathfrak{s}}\right\} / \hat{\mathcal{G}} .
$$

and this is identified with the $I$-invariant moduli space,

$$
\mathcal{M}(X, \mathfrak{s})^{I}=\{\text { Seiberg-Witten solutions on } \mathfrak{s}\}^{I} / \mathcal{G}^{I} .
$$

By Proposition 2.13, we have the identifications,

$$
\hat{\mathcal{M}}(\hat{X}, \hat{\mathfrak{s}}) \cong \mathcal{M}(X, \mathfrak{s})^{I} \cong \mathcal{M}(X, \overline{\mathfrak{s}})^{I} \cong \hat{\mathcal{M}}\left(\hat{X}, \hat{\mathfrak{s}}^{c}\right) .
$$

The second identification is the isomorphism of complex conjugation in the ordinary Seiberg-Witten theory.

Corollary 2.14. $\mathrm{SW}^{\mathrm{Pin}}\left(\hat{X}, \hat{\mathfrak{s}}^{c}\right)= \pm \mathrm{SW}^{\operatorname{Pin}}(\hat{X}, \hat{\mathfrak{s}})$.

\section{Real symplectic 4-manifolds}

In this section, we prove Theorem 1.1 and Theorem 1.4. Suppose a closed real symplectic 4-manifold $(X, \omega, \iota)$ satisfies the assumption of Theorem 1.1 .

First we consider the $\mathrm{Pin}^{-}(2)$-monopole equations on the canonical Spin ${ }^{c_{-}}$ structure. Let $\mathfrak{s}_{0}$ be the canonical Spin ${ }^{c}$ structure on $(X, \omega)$ and $\hat{\mathfrak{s}}_{0}$ the canonical Spin ${ }^{c_{-}}$structure on $X \rightarrow \hat{X}$. Recall $\hat{\omega}$ is a $\ell_{\mathbb{R}^{-v a l u e d ~ s e l f-d u a l ~}}$ 
2 -form such that $\omega=\pi^{*} \hat{\omega}$. Normalize the metric on $X$ so that $|\hat{\omega}|=\sqrt{2}$ and pull it back to $\hat{X}$ so that $|\omega|=\sqrt{2}$. Recall the splitting

$$
\Lambda^{+}(X) \otimes_{\mathbb{R}} \mathbb{C}=\mathbb{C} \cdot \omega \oplus K \oplus K^{-1} .
$$

The Clifford multiplication by $\omega$ induces the splitting $W_{0}^{+}=\underline{\mathbb{C}} \oplus K^{-1}$. In fact, the components $\underline{\mathbb{C}}$ and $K^{-1}$ are respectively $(+2)$ and (-2)-eigenspaces of the action of $(\omega / i)$ on $W_{0}^{+}$.

On the $\operatorname{Spin}^{c_{-}}$structure $\hat{\mathfrak{s}}_{0}$, we have a twisted Clifford multiplication $\rho: \Lambda^{1}(\hat{X}) \otimes i \ell_{\mathbb{R}} \rightarrow \operatorname{Hom}\left(\hat{W}_{0}^{+}, \hat{W}_{0}^{-}\right)$[17], and this extends to

$$
\rho:: \Lambda^{+}(\hat{X}) \otimes i \ell_{\mathbb{R}} \rightarrow \operatorname{End}\left(\hat{W}_{0}^{+}\right) .
$$

Then $(\hat{\omega} / i)$ induces the splitting $\hat{W}_{0}^{+}=\underline{\hat{\mathbb{C}}} \oplus \hat{K}^{-1}$. Since the real part of $\underline{\hat{\mathbb{C}}}$ is trivial, there is a constant section $\hat{u}_{0}$ such that $\left|\hat{u}_{0}\right|=1$. Mimicking the argument of Taubes [23], we obtain the following.

Proposition 3.1. There is a unique $\mathrm{O}(2)$-connection $\hat{A}_{0}$ (up to gauge) on $\hat{P}_{K}$ whose induced covariant derivative $\nabla_{\hat{A}_{0}}$ on $\hat{W}^{+}$has the property that

$$
\left(1+\frac{1}{2} \rho(\hat{\omega} / i)\right) \nabla_{\hat{A}_{0}} \hat{u}_{0}=0
$$

Furthermore, $D_{\hat{A}_{0}} \hat{u}_{0}=0$ if and only if $d \hat{\omega}=0$, where $D_{\hat{A}_{0}}$ is the Dirac operator associated with $\hat{A}_{0}$.

Let us consider the $\mathrm{Pin}^{-}(2)$-monopole equations rescaled and perturbed as follows:

$$
D_{\hat{A}} \hat{\phi}=0, \quad F_{\hat{A}}^{+}=r q(\hat{\phi})-\frac{r}{4} i \hat{\omega}+F_{\hat{A}_{0}}^{+},
$$

where $\hat{A}$ is an $\mathrm{O}(2)$-connection on $\hat{P}_{K}, \hat{\phi} \in \Gamma\left(\hat{W}_{0}^{+}\right), q$ is the quadratic form defined in [17] and $r$ is a positive real constant. (This is an analogue of Taubes' perturbation [25].) Then we can see that $\left(\hat{A}_{0}, \hat{u}_{0}\right)$ is a solution to (3.2) for every $r$.

To proceed further, it is convenient to move to the upstairs and consider the $I$-invariant part. Let $\left(A_{0}, u_{0}\right)$ be the lift of $\left(\hat{A}_{0}, \hat{u}_{0}\right)$. Then a spinor $\phi \in \Gamma\left(W_{0}^{+}\right)$can be written as $\phi=\alpha u_{0}+\beta$, where $\alpha$ is a complex-valued function on $X$ and $\beta \in \Gamma\left(K^{-1}\right)$. Then a solution to the equation (3.2) corresponds to an $I$-invariant solution to the perturbed equation due to Taubes [25]:

$$
\begin{aligned}
D_{A} \phi & =0 \\
F_{A}^{+}-F_{A_{0}}^{+} & =-\frac{i r}{8}\left(1-|\alpha|^{2}+|\beta|^{2}\right) \omega+\frac{i r}{4}(\alpha \bar{\beta}+\bar{\alpha} \beta) .
\end{aligned}
$$

Proof of Theorem 1.1. By construction, $\left(A_{0}, u_{0}\right)$ is an $I$-invariant solution to (3.3) for every $r$. Taubes [23 25] (see also Kotschick [14]) proved that there is no solution to (3.3) except $\left(A_{0}, u_{0}\right)$ for large $r$. It follows from this that $\left(\hat{A}_{0}, \hat{u}_{0}\right)$ is a unique solution to (3.2) for large $r$. These imply Theorem 1.1 .

Proof of Theorem 1.4. Suppose the $\mathrm{Pin}^{-}(2)$-monopole invariant on a $\mathrm{Spin}^{c_{-}}$ structure $\hat{\mathfrak{s}}$ is nonzero. Then the equations (3.2) on $\hat{\mathfrak{s}}$ has a solution $(\hat{A}, \hat{\phi})$ for every $r$. Then the lift $(A, \phi)$ of $(\hat{A}, \hat{\phi})$ is an $I$-invariant solution to (3.3) 
for $r$. By Kotschick [14](Cf. Taubes [24]), the existence of solutions for large $r$ implies that

$$
\left|c_{1}(L) \cdot[\omega]\right| \leq c_{1}(K) \cdot[\omega],
$$

where $L$ is the determinant line bundle of $(X, \mathfrak{s})$. Let $\hat{L}$ be the characteristic bundle for $(\hat{X}, \hat{\mathfrak{s}})$. Then $L=\pi^{*} \hat{L}$. The inequality (1.5) follows from (3.4)

By 25, we can find an embedded symplectic curve $C$ in $X$ such that $e=P . D .[C]$ satisfies $e^{2}=c_{1}(K \otimes L)$. If $X$ contains embedded 2-spheres with self-intersection number -1 , then blow them down. The resulting manifold is a minimal symplectic manifold $X^{\prime}$ with another embedded symplectic curve $C^{\prime}$ (see, e.g., [20]). Then the proof of Theorem 0.2(6) of [25] implies that the virtual dimension $d(\mathfrak{s})$ of the moduli space for $(X, \mathfrak{s})$ is 0 . Therefore $d(\hat{\mathfrak{s}})=\frac{1}{2} d(\mathfrak{s})=0$.

\section{Real Kähler surfaces}

The purpose of this section is to prove that the $\mathrm{Pin}^{-}(2)$-monopole moduli space on a real Kähler surface can be identified with the $I$-invariant moduli space of holomorphic simple pairs, and the space of $I$-invariant effective divisors. The moduli space of vortices is also introduced as an intermediate object. The goal of this section is Corollary 4.22 and Corollary 4.26 ,

Let $(X, \omega, \iota)$ be a compact Kähler surface with anti-holomorphic free involution $\iota$ such that $\iota^{*} \omega=-\omega$. Note that the pull-back of a $(p, q)$-form by the anti-linear map $\iota$ is a $(q, p)$-form, and the complex conjugation of a $(q, p)$-form is a $(p, q)$-form. We define the involution $I$ on the space of $(p, q)$-forms $\Omega^{p, q}(X)$ by

$$
I(\alpha)=\overline{\iota^{*} \alpha}, \quad \alpha \in \Omega^{p, q}(X) .
$$

Note that $K=\Lambda^{2,0}(X), K^{-1}=\Lambda^{0,2}(X), \iota^{*} K=K^{-1}$.

Suppose that there is a canonical $\mathrm{Spin}^{c_{-}}$structure $\hat{\mathfrak{s}}_{0}$ on $X \rightarrow \hat{X}=X / \iota$. As explained in $\$ 2.2$, every $\operatorname{Spin}^{c_{-}}$structure on $X \rightarrow \hat{X}$ is obtained from $\hat{\mathfrak{s}}_{0}$ and an $\mathbb{R}^{2}$-bundle $\hat{E}$ twisted along $\ell_{\mathbb{R}}$ as $\hat{\mathfrak{s}}_{0} \hat{\otimes} \hat{E}$.

For a $\operatorname{Spin}^{c-}$ structure $\hat{\mathfrak{s}}=\hat{\mathfrak{s}}_{0} \hat{\otimes} \hat{E}$, there exists a $\operatorname{Spin}^{c}$ structure $\mathfrak{s}=\mathfrak{s}_{0} \otimes E$ on $X$ which is the canonical reduction of $\pi^{*} \hat{\mathfrak{s}}$, whose positive spinor bundle is $W^{+}=E \oplus\left(E \otimes K^{-1}\right)$ such that $E \cong \pi^{*} \hat{E}$ as $\mathbb{R}^{2}$-bundles. Note that $\iota^{*} \mathfrak{s}=\overline{\mathfrak{s}}$. Then $\iota^{*} E=\bar{E}$ and $E$ naturally admits a Hermitian metric $h$ such that $\iota^{*} h=\bar{h}$.

Let $C$ be a Hermitian connection on $K^{-1}$ induced by the Chern connection on $T X$ associated with the Kähler structure.

Recall that the Dirac operator $D$ on the canonical $\operatorname{Spin}^{c}$ structure $\mathfrak{s}_{0}$ is identified with

$$
D=\sqrt{2}\left(\bar{\partial}+\bar{\partial}^{*}\right): \Omega^{0,0}(X) \oplus \Omega^{0,2}(X) \rightarrow \Omega^{0,1}(X) .
$$

Since $\iota$ is anti-holomorphic, the pull-back of $D$ by $\iota$ is

$$
\iota^{*} D=\sqrt{2}\left(\partial+\partial^{*}\right): \Omega^{0,0}(X) \oplus \Omega^{2,0}(X) \rightarrow \Omega^{1,0}(X) .
$$

Then we see that the Dirac operator $D$ is $I$-equivariant.

Next we consider Dirac operators on a $\operatorname{Spin}^{c}$ structure $\mathfrak{s}=\mathfrak{s}_{0} \otimes E$. For a Hermitian connection $A$ on $\operatorname{det}\left(W^{+}\right)=E^{2} \otimes K^{-1}$, there is a unique 
Hermitian connection $B$ on $E$ such that $A=C \otimes B^{\otimes 2}$. Then the Dirac operator $D_{A}$ associated with $A$ is identified with

$$
D_{A}=\sqrt{2}\left(\bar{\partial}_{B}+\bar{\partial}_{B}^{*}\right): \Omega^{0,0}(E) \oplus \Omega^{0,2}(E) \rightarrow \Omega^{0,1}(E) .
$$

The pull-back $B^{\prime}=\iota^{*} B$ is a Hermitian connection on $\bar{E}=\iota^{*} E$, and the pull-back $\iota^{*} D_{A}$ can be written as

$$
\iota^{*} D_{A}=\sqrt{2}\left(\partial_{B^{\prime}}+\partial_{B^{\prime}}^{*}\right): \Omega^{0,0}(\bar{E}) \oplus \Omega^{2,0}(\bar{E}) \rightarrow \Omega^{1,0}(\bar{E}) .
$$

For an $\mathrm{O}(2)$-connection $\hat{B}$ on $\hat{E}$, we have a Hermitian connection $B$ on $E$ which is the $\mathrm{U}(1)$-reduction of $\pi^{*} \hat{B}$. Then $B$ is $I$-invariant, i.e., $B=\overline{\iota^{*} B}$. For such a connection $B$, the Dirac operator $D_{A}=\sqrt{2}\left(\bar{\partial}_{B}+\bar{\partial}_{B}^{*}\right)$ is also I-equivariant.

Recall the identifications:

$$
H^{2}(X ; \mathbb{C})=H^{1,1} \oplus H^{2,0} \oplus H^{0,2}, \quad H_{+}(X ; i \mathbb{R})=i \mathbb{R} \omega \oplus H^{0,2} .
$$

Note that the $I=\overline{\iota^{*}(\cdot)}$-action preserves $H_{+}(X ; i \mathbb{R})$ and

$$
H_{+}\left(\hat{X} ; \ell_{\mathbb{R}}\right) \cong H_{+}(X ; i \mathbb{R})^{I}=i \mathbb{R} \omega \oplus\left(H^{0,2}\right)^{I} .
$$

In particular, we have the following.

Proposition 4.1. If $b_{+}^{\ell}=\operatorname{rank} H_{+}\left(\hat{X} ; \ell_{\mathbb{R}}\right) \geq 2$, then $\left(H^{2,0}\right)^{I} \cong\left(H^{0,2}\right)^{I} \neq \emptyset$.

4.1. Seiberg-Witten equations. The Seiberg-Witten equations on Kähler surfaces can be written as follows([16, 27]):

$$
\begin{aligned}
\bar{\partial}_{B} \alpha+\bar{\partial}_{B} \beta & =0 \\
2 F_{B}^{0,2}+2 \pi i \eta^{0,2}-\frac{1}{2} \beta \bar{\alpha} & =0 \\
2 F_{B}^{2,0}+2 \pi i \eta^{2,0}+\frac{1}{2} \alpha \bar{\beta} & =0 \\
\left\{\Lambda_{g}\left(F_{B}+\pi i \eta\right)-\frac{i}{2} s_{g}+\frac{i}{8}\left(|\beta|^{2}-|\alpha|^{2}\right)\right\} \omega & =0
\end{aligned}
$$

These are the equations for Hermitian connections $B$ on $E$ and sections $(\alpha, \beta) \in\left(\Omega^{0,0} \oplus \Omega^{0,2}\right)(E)$. The perturbation term is given by $\eta \in \Omega^{2}(X), \Lambda_{g}$ denotes the adjoint of the multiplication operator $\omega \wedge \cdot: i \Omega^{0,0} \rightarrow i \Omega^{1,1}$, and $s_{g}$ is the scalar curvature. (Here we use the fact that $i \Lambda_{g} F_{C}=s_{g}$ for the Chern connection $C$.) If we take an $I$-invariant $\eta$, then (4.2) is $I$-equivariant.

The discussion below is largely indebted to Teleman's excellent exposition [27]. The general principle is to "consider in the upstairs and take the $I$-invariant part". The next two theorems are obtained by restricting everything to the $I$-invariant part in the corresponding theorems of [27].

Theorem 4.3 ([27], Théorème 8.1.7). Suppose $\eta$ is an I-invariant closed $(1,1)$-form, and

$$
\Theta:=\frac{1}{2}\left\langle\left([\eta]-2 c_{1}(E)+c_{1}(K)\right) \cup[\omega],[X]\right\rangle \neq 0
$$

Then an I-invariant triple $(B, \alpha, \beta)$ is a solution to (4.2) if and only if: I. $\Theta>0$ and

$$
\beta=0, \quad \bar{\partial}_{B} \alpha=0, \quad F_{B}^{0,2}=0, \quad i \Lambda_{g} F_{B}+\frac{1}{8}|\alpha|^{2}=\pi \Lambda_{g} \eta-\frac{s_{g}}{2}
$$


II. $\Theta<0$ and

$$
\alpha=0, \quad \bar{\partial}_{B}^{*} \beta=0, \quad F_{B}^{0,2}=0, \quad i \Lambda_{g} F_{B}-\frac{1}{8}|\beta|^{2}=\pi \Lambda_{g} \eta-\frac{s_{g}}{2}
$$

Let $C^{\vee}$ be the Hermitian connection on $K$ induced from the Chern connection $C$. For a Hermitian connection on $E$, let $B^{\prime}$ be the Hermitian connection on $K \otimes \bar{E}$ such that $B \otimes B^{\prime}=C^{\vee}$. For $\beta \in \Omega^{0,2}(E)=\Gamma\left(E \otimes K^{-1}\right)$, let $\varphi=\bar{\beta} \in \Gamma(\bar{E} \otimes K)$. Then the condition $\bar{\partial}_{B}^{*} \beta=0$ is equivalent to $\bar{\partial}_{B^{\prime}} \varphi=0$ by the Serre duality, and (4.5) can be rewritten as

$$
\alpha=0, \quad \bar{\partial}_{B^{\prime}} \varphi=0, \quad F_{B^{\prime}}^{0,2}=0, \quad i \Lambda_{g} F_{B^{\prime}}+\frac{1}{8}|\varphi|^{2}=-\pi \Lambda_{g} \eta+\frac{s_{g}}{2}
$$

If $\eta$ is not a $(1,1)$-form, then we have the following.

Theorem 4.6 ([27], Théorème 9.3.1). Suppose an I-invariant 2-form $\eta$ has a form of $\eta=\eta^{2,0} \oplus \eta^{1,1} \oplus \overline{\eta^{2,0}}$ where $\eta^{2,0}$ is an I-invariant non-zero holomorphic 2-form. Then an I-invariant triple $(B, \alpha, \beta)$ is a solution to (4.2) if and only if:

$$
\begin{gathered}
\alpha \bar{\beta}=-8 \pi i \eta^{2,0}, \quad \bar{\partial}_{B} \alpha=\bar{\partial}_{B}^{*} \beta=0, \quad F_{B}^{0,2}=0, \\
i \Lambda_{g} F_{B}+\frac{1}{8}\left(|\beta|^{2}-|\alpha|^{2}\right)=\pi \Lambda_{g} \eta^{1,1}-\frac{s_{g}}{2}
\end{gathered}
$$

Let $C^{\vee}, B^{\prime}$ and $\varphi$ be as above. Then (4.7) can be rewritten as

$$
\begin{gathered}
\alpha \varphi=-8 \pi i \eta^{2,0}, \quad B \otimes B^{\prime}=C^{\vee}, \\
\bar{\partial}_{B} \alpha=\bar{\partial}_{B^{\prime}} \varphi=0, \quad F_{B}^{0,2}=F_{B^{\prime}}^{0,2}=0, \\
\frac{i}{2} \Lambda_{g}\left(F_{B}-F_{B^{\prime}}\right)+\frac{1}{8}\left(|\varphi|^{2}-|\alpha|^{2}\right)=\pi \Lambda_{g} \eta
\end{gathered}
$$

4.2. Vortex equations. Let $(X, \omega, \iota)$ be a compact Kähler surface with anti-holomorphic free involution $\iota$. Suppose we have a $C^{\infty}$ Hermitian line bundle $(E, h)$ over $X$ with an isomorphism $\iota^{*}(E, h) \cong(\bar{E}, \bar{h})$. This isomorphism defines the bundle map $I=\overline{\iota^{*}(\cdot)}$ covering $\iota$ which is the composite map of

$$
E \stackrel{\iota^{*}}{\longrightarrow} \iota^{*} E \cong \bar{E} \stackrel{(\bar{\cdot})}{\longrightarrow} E .
$$

We suppose $I$ generates an order-2 action (involution) on $E$. We define the $I$-action on $\Omega^{0}(E)$ also by $I=\overline{\iota^{*}(\cdot)}$. Let $\mathcal{A}(E, h)$ be the space of Hermitian connections on $E$. Then the involution $I$ naturally induces an involution on $\mathcal{A}(E, h)$, also denoted by $I$. The gauge transformation group $\mathcal{G}=C^{\infty}\left(X ; S^{1}\right)$ acts on $\mathcal{A}(E, h) \times \Omega^{0}(E)$ by

$$
(B, \phi) \cdot f=\left(B+f^{-1} d f, f^{-1} \phi\right) \text { for }(B, \phi) \in \mathcal{A}(E, h) \times \Omega^{0}(E), f \in \mathcal{G} .
$$

A configuration $(B, \phi)$ with $\phi \neq 0$ is called an irreducible. The group $\mathcal{G}$ acts on the space of irreducibles freely. We define the involution $I$ on $\mathcal{G}$ by $I(f)=\overline{\iota^{*} f}$. Then the $\mathcal{G}$-action on $\mathcal{A}(E, h) \times \Omega^{0}(E)$ is $I$-equivariant. 
Definition 4.9. Let $t: X \rightarrow \mathbb{R}$ be a $C^{\infty}$-function. A $t$-vortex is a solution $(B, \phi) \in \mathcal{A}(E, h) \times \Omega^{0}(E)$ to the system of the equations

$$
\begin{gathered}
\bar{\partial}_{B} \phi=0 \\
F_{B}^{0,2}=0 \\
i \Lambda_{g} F_{B}+\frac{1}{2}|\phi|^{2}-t=0
\end{gathered}
$$

If $(B, \phi)$ is a solution to (4.10), then

$$
\Xi:=\frac{1}{2 \pi} \int_{X} t d v o l_{g}-\left\langle c_{1}(E) \cup[\omega],[X]\right\rangle=\frac{1}{2 \pi} \int_{X}\left(t-i \Lambda_{g} F_{B}\right) d v o l_{g}=\frac{1}{4 \pi}\|\phi\|_{L^{2}}^{2} \geq 0 .
$$

If $t$ is $\iota$-invariant, that is, $\iota^{*} t=t$, then the system (4.10) is $I$-equivariant. Define $I$-invariant moduli spaces as follows:

$$
\begin{aligned}
\mathcal{V}_{t}(E)^{I} & =\{I \text {-invariant } t \text {-vortices }\} / \mathcal{G}^{I}, \\
\mathcal{V}_{t}^{*}(E)^{I} & =\{I \text {-invariant irreducible } t \text {-vortices }\} / \mathcal{G}^{I} .
\end{aligned}
$$

If $\Xi>0$, then $\mathcal{V}_{t}^{*}(E)^{I}=\mathcal{V}_{t}(E)^{I}$.

As usual, we take $L_{k}^{2}$-completion of $\mathcal{C}^{*}(E):=\mathcal{A}(E, h) \times\left(\Omega^{0}(E) \backslash\{0\}\right)$ and $L_{k+1}^{2}$-completion of $\mathcal{G}$ for sufficiently large $k$. We use the notation $(\cdot)_{k}$ for the completed spaces. For a generic choice of $I$-invariant $t$ with positive $\Xi$, the space $\mathcal{V}_{t}^{*}(E)^{I}$ is a submanifold of the Hilbert manifold

$$
\left(\mathcal{B}_{k}^{*}\right)^{I}:=\left(\mathcal{C}^{*}(E)_{k}\right)^{I} / \mathcal{G}_{k+1}^{I} .
$$

For an orbit $[v]=[(B, \phi)] \in\left(\mathcal{B}_{k}^{*}\right)^{I}$, the tangent space of $\left(\mathcal{B}_{k}^{*}\right)^{I}$ at $[v]$ is given by

$$
T_{[v]}\left(\mathcal{B}_{k}^{*}\right)^{I}=\left\{(\dot{B}, \dot{\phi}) \mid d^{*} \dot{B}-i \operatorname{Im}(\dot{\phi} \bar{\phi})=0\right\}^{I} .
$$

The following is a direct consequence of Theorem 4.3 ,

Corollary 4.12. Suppose $\eta$ is an I-invariant closed $(1,1)$-form. Let $t=$ $\pi \Lambda_{g} \eta-s_{g} / 2$. Then we have the following identifications:

(1) $\mathcal{M}\left(X, \mathfrak{s}_{0} \otimes E\right)^{I} \cong \mathcal{V}_{t}^{*}(E)^{I}$, if $\Theta>0$.

(2) $\mathcal{M}\left(X, \mathfrak{s}_{0} \otimes E\right)^{I} \cong \mathcal{V}_{-t}^{*}\left(K \otimes E^{-1}\right)^{I}$, if $\Theta<0$.

4.3. Holomorphic simple pairs. Let $(X, \omega, \iota)$ be a compact Kähler surface with anti-holomorphic involution $\iota$, and $E$ a $C^{\infty}$ complex line bundle such that $\iota^{*} E \cong \bar{E}$. As before, we suppose $I=\overline{\iota^{*}(\cdot)}$ generates an involution on $E$. We define the $I$-action on $\Omega^{0}(E)$ also by $I=\overline{\iota^{*}(\cdot)}$. Let $\mathcal{A}^{0,1}(E)$ be the space of semiconnections on $E$. Note that a semiconnection $\delta \in \mathcal{A}^{0,1}(E)$ can be written as $\delta=\bar{\partial}_{B}$ for some complex linear connection $B$ on $E$. The involution $I$ naturally induces an involution on $\mathcal{A}^{0,1}(E)$, also denoted by $I$. The complex gauge transformation group $\mathcal{G}^{\mathbb{C}}=C^{\infty}\left(X, \mathbb{C}^{*}\right)$ acts on $\mathcal{P}(E)=\mathcal{A}^{0,1}(E) \times \Omega^{0}(E)$ by

$$
(\delta, \phi) \cdot f=\left(\delta \cdot f, f^{-1} \phi\right) \text { for }(\delta, \phi) \in \mathcal{P}(E), \quad f \in \mathcal{G}^{\mathbb{C}},
$$

where $\delta \cdot f=f^{-1} \circ \delta \circ f=\delta+f^{-1} \bar{\partial} f$. A pair $(\delta, \phi)$ with nonzero $\phi$ is called simple. Let $\mathcal{P}^{s}(E)$ be the space of simple pairs. Then $\mathcal{G}^{\mathbb{C}}$ acts on $\mathcal{P}^{s}(E)$ freely. We define the involution $I$ on $\mathcal{G}^{\mathbb{C}}$ by $I(f)=\overline{\iota^{*} f}$. Then the $\mathcal{G}^{\mathbb{C}}$-action on $\mathcal{P}(E)$ is $I$-equivariant. 
Let $\mathcal{H}(E)$ be the space of holomorphic pairs:

$$
\mathcal{H}(E)=\left\{(\delta, \phi) \in \mathcal{A}^{0,1}(E) \times \Omega^{0}(E) \mid \delta \circ \delta=0, \delta \phi=0\right\} .
$$

A pair $(\delta, \phi) \in \mathcal{H}(E)$ with non-zero $\phi$ is called a holomorphic simple pair. Let $\mathcal{H}^{s}(E)$ be the space of holomorphic simple pairs.

We consider the $I$-invariant moduli space of holomorphic simple pairs:

$$
\mathcal{M}^{s}(E)^{I}=\mathcal{H}^{s}(E)^{I} /\left(\mathcal{G}^{\mathbb{C}}\right)^{I} .
$$

The deformation complex for an $I$-invariant holomorphic simple pair $\mathfrak{p}=$ $(\delta, \phi)$ is given by

$$
\left(\mathcal{C}_{\mathfrak{p}}\right)^{I}=\left(\mathcal{C}^{0}\right)^{I} \stackrel{\mathcal{D}_{\mathfrak{p}}^{0}}{\rightarrow}\left(\mathcal{C}^{1}\right)^{I} \stackrel{\mathcal{D}_{\mathfrak{p}}^{1}}{\rightarrow}\left(\mathcal{C}^{2}\right)^{I} \stackrel{\mathcal{D}_{\mathfrak{p}}^{2}}{\rightarrow}\left(\mathcal{C}^{3}\right)^{I},
$$

where

$$
\begin{array}{cc}
\mathcal{C}^{0}=\Omega^{0,0}(X), & \mathcal{C}^{i}=\Omega^{0, i}(X) \oplus \Omega^{0, i-1}(E)(i=1,2), \quad \mathcal{C}^{3}=\Omega^{0,2}(E), \\
\mathcal{D}_{\mathfrak{p}}^{i}(\alpha, \sigma) & =(\bar{\partial} \alpha,-\delta \sigma-\alpha \phi) .
\end{array}
$$

The moduli space $\mathcal{M}^{s}(E)^{I}$ has a Kuranishi model as follows.

Proposition 4.15 (Cf. [27], Proposition 8.2.10). Let $H^{i}\left(\left(\mathcal{C}_{\mathfrak{p}}\right)^{I}\right), \mathbb{H}^{i}\left(\left(\mathcal{C}_{\mathfrak{p}}\right)^{I}\right)$ be the cohomology group and harmonic space of the elliptic complex $\left(\mathcal{C}_{\mathfrak{p}}\right)^{I}$. There exists a neighborhood $U_{\mathfrak{p}}$ of $0 \in \mathbb{H}^{1}\left(\left(\mathcal{C}_{\mathfrak{p}}\right)^{I}\right)$ and a smooth map

$$
\mathfrak{t}_{\mathfrak{p}}: U_{\mathfrak{p}} \rightarrow \mathbb{H}^{2}\left(\left(\mathcal{C}_{\mathfrak{p}}\right)^{I}\right)
$$

such that a neighborhood of $\mathfrak{p} \in \mathcal{M}^{s}(E)^{I}$ is homeomorphic to $\mathfrak{t}_{\mathfrak{p}}^{-1}(0)$. Furthermore, if $H^{2}\left(\left(\mathcal{C}_{\mathfrak{p}}\right)^{I}\right)=0$, then $\mathcal{M}^{s}(E)^{I}$ is a smooth manifold of dimension $\operatorname{dim} H^{1}\left(\left(\mathcal{C}_{\mathfrak{p}}\right)^{I}\right)$ near $[\mathfrak{p}]$, and the tangent space of $\mathcal{M}^{s}(E)^{I}$ at $[\mathfrak{p}]$ is identified with $H^{1}\left(\left(\mathfrak{C}_{\mathfrak{p}}\right)^{I}\right)$.

The proof is standard.

4.4. I-invariant divisors. (A reference of this subsection is [21], I.4.) A Weil divisor is a formal linear combination $\sum_{i} n_{i} D_{i}$ of irreducible analytic hypersurfaces. Define the $I$-action on divisors by $I \cdot D=\sum_{i} n_{i} \iota\left(D_{i}\right)$. We call a divisor $D I$-invariant if $D=I \cdot D$. We will mainly consider effective divisors, i.e., $D=\sum_{i} n_{i} D_{i}$ with $n_{i} \geq 0$.

When $D$ is considered as a Cartier divisor, the $I$-action can be written as follows. For an open subset $U \subset X$ and a holomorphic function $f \in \mathcal{O}_{X}(U)$, define $I \cdot f \in \mathcal{O}_{X}(\iota(U))$ by $(I \cdot f)(x)=\overline{f(\iota x)}$. Let $\mathcal{S}$ be the set of pairs $\left(U_{\lambda}, \lambda\right)$ where $U_{\lambda}$ is an open set and $\lambda \in \mathcal{O}_{X}\left(U_{\lambda}\right)$. Then define the $I$-action on $\mathcal{S}$ by

$$
I \cdot\left(U_{\lambda}, \lambda\right)=\left(\iota\left(U_{\lambda}\right), I \cdot \lambda\right)
$$

An effective Cartier divisor is given as a subset $\mathcal{F} \subset \mathcal{S}$ whose elements $\left(U_{\lambda}, \lambda\right)$ satisfy the following:

(1) $\lambda$ is not identically zero.

(2) $\bigcup_{\lambda \in \mathcal{F}} U_{\lambda}=X$.

(3) For every $\lambda, \mu \in \mathcal{F}$, there exists $g_{\lambda, \mu} \in \mathcal{O}_{X}^{*}\left(U_{\lambda} \cap U_{\mu}\right)$ such that $\lambda=$ $g_{\lambda, \mu} \mu$. 
We will take a maximal one of such systems for $\mathcal{F}$. The effective Weil divisor corresponding to an effective Cartier divisor is obtained by considering $\lambda$ as local defining equations. Let

$$
I \cdot \mathcal{F}=\left\{I \cdot\left(U_{\lambda}, \lambda\right) \mid\left(U_{\lambda}, \lambda\right) \in \mathcal{F}\right\} .
$$

Then $I \cdot D$ corresponds to $I \cdot \mathcal{F}$. Note that $g_{I \lambda, I \mu}=I g_{\lambda, \mu}$. If $D$ is $I$-invariant, then we can take $\mathcal{F}$ corresponding to $D$ such that $\mathcal{F}=I \cdot \mathcal{F}$.

The system of cocycles $\left\{g_{\lambda, \mu}\right\}$ and local functions $\{\lambda\}$ defines a holomorphic line bundle $\mathcal{L}$ with a holomorphic section $\phi$ as follows.

$$
\begin{gathered}
\mathcal{L}=\left(\bigcup_{\lambda \in \mathcal{F}}\{\lambda\} \times U_{\lambda} \times \mathbb{C}\right) / \sim, \\
\{\mu\} \times\left(U_{\lambda} \cap U_{\mu}\right) \times \mathbb{C} \ni(\mu, u, \zeta) \sim\left(\lambda, u, g_{\lambda, \mu} \zeta\right) \in\{\lambda\} \times\left(U_{\lambda} \cap U_{\mu}\right) \times \mathbb{C}, \\
\phi(u)=[(\lambda, u, \lambda(u))] \bmod \sim\left(u \in U_{\lambda}\right) .
\end{gathered}
$$

Then the corresponding divisor $D$ is $D=Z(\phi)$. When $\left(\mathcal{L}_{D}, \phi_{D}\right)$ is associated with $D$ (or $\mathcal{F}$ ), note that the line bundle with section associated with $I \cdot D$ (or $I \cdot \mathcal{F}$ ) is

$$
\left(\mathcal{L}_{I \cdot D}, \phi_{I \cdot D}\right)=\left(\overline{\iota^{*} \mathcal{L}_{D}}, \overline{\iota^{*} \phi_{D}}\right) .
$$

If $D$ is $I$-invariant, then an anti-linear involution $I$ on $\mathcal{L}_{D}$ covering $\iota$ is naturally defined by

$$
I \cdot[(\lambda, u, \zeta)]=[(I \lambda, \iota(u), \zeta)] .
$$

4.5. $I$-equivariant sheaves. Under the $I$-action, the structure sheaf $\mathcal{O}_{X}$ is an $I$-equivariant sheaf in the sense of [10,22], i.e., the sheaf projection $\mathcal{O}_{X} \rightarrow X$ is $I$-equivariant. If $D$ is $I$-invariant, then $\mathcal{O}_{X}(D)$ and $\mathcal{O}_{D}(D)=$ $\mathcal{O}_{X}(D) / \mathcal{O}_{X}$ are also $I$-equivariant. For an $I$-equivariant sheaf $\mathcal{E}$, the equivariant sheaf cohomology $H^{p}(X ; I, \mathcal{E})$ is defined: For an $I$-invariant open set $U \subset X$, let $\Gamma^{I}(U ; \mathcal{E})$ be the module of $I$-invariant sections. Take an injective resolution $\mathcal{J}^{*}(\mathcal{E})$ of $\mathcal{E}$ in the category of $I$-equivariant sheaves. Then $H^{p}(X ; I, \mathcal{E})$ is defined by

$$
H^{p}(X ; I, \mathcal{E})=H^{p}\left(\Gamma^{I}\left(X ; \mathcal{J}^{*}(\mathcal{E})\right) .\right.
$$

The equivariant direct image $\pi^{I} \mathcal{E}$ of $\mathcal{E}$ is the sheaf on $\hat{X}=X / \iota$ which is generated by the presheaf,

$$
\hat{U} \mapsto \Gamma^{I}\left(\pi^{-1}(\hat{U}) ; \mathcal{E}\right), \quad \hat{U} \subset \hat{X}=X / \iota \quad \text { open. }
$$

In general, $\pi^{G}$ is a left exact functor for $G$-sheaves. However our case is much simple. Since $I$ covers the free involution $\iota$ on $X, \pi^{I}$ is an exact functor. That is, for an exact sequence of $I$-sheaves on $X$,

$$
0 \rightarrow \mathcal{E} \rightarrow \mathcal{F} \rightarrow \mathcal{H} \rightarrow 0,
$$

we have an exact sequence of sheaves on $\hat{X}$,

$$
0 \rightarrow \pi^{I} \mathcal{E} \rightarrow \pi^{I} \mathcal{F} \rightarrow \pi^{I} \mathcal{H} \rightarrow 0 .
$$

In particular,

$$
\pi^{I} \mathcal{F} / \pi^{I} \mathcal{E}=\pi^{I}(\mathcal{F} / \mathcal{E}) .
$$

The fact that $I$ covers the free involution $\iota$ on $X$ also implies that

$$
H^{i}(X ; I, \mathcal{E})=H^{i}\left(X / \iota ; \pi^{I}(\mathcal{E})\right) .
$$


(10], p.204, Corollaire; 22, Corollary 5.6.)

There is an $I$-equivariant exact sequence.

$$
0 \longrightarrow \tilde{\mathbb{Z}} \stackrel{i}{\longrightarrow} \mathcal{O}_{X} \stackrel{\exp 2 \pi}{\longrightarrow} \mathcal{O}_{X}^{*} \longrightarrow 0,
$$

where $\tilde{\mathbb{Z}}$ is the constant sheaf on which $I$ acts via multiplication of -1 . This induces the sequence

$$
0 \rightarrow H^{1}(X ; I, \tilde{\mathbb{Z}}) \rightarrow H^{1}\left(X ; I, \mathcal{O}_{X}\right) \rightarrow H^{1}\left(X ; I, \mathcal{O}_{X}^{*} \stackrel{\stackrel{\tilde{c}_{1}}{\rightarrow}}{\rightarrow} H^{2}(X ; I, \tilde{\mathbb{Z}}) \rightarrow \cdots .\right.
$$

Note that $H^{i}(X ; I, \tilde{\mathbb{Z}}) \cong H^{i}(\hat{X} ; \ell)$. Let $\mathrm{NS}^{I}(X)=\operatorname{Im} \tilde{c}_{1}$. For $e \in \operatorname{NS}^{I}(X)$, let $\mathcal{D}(e)$ be the set of effective divisors representing $e$.

Proposition 4.16 ([27], Proposition 8.2.13). Let $e=\tilde{c}_{1}(E)$.

(1) The map $(\delta, \phi) \mapsto Z(\phi)$ induces a bijection $\mathcal{M}^{s}(E)^{I} \cong \mathcal{D}(e)^{I}$.

$$
H^{0}\left(\left(\mathcal{C}_{\mathfrak{p}}\right)^{I}\right)=0, \quad H^{i}\left(\left(\mathcal{C}_{\mathfrak{p}}\right)^{I}\right) \cong H^{i-1}\left(X ; I, \mathcal{O}_{D}(D)\right)=H^{i-1}\left(\hat{X} ; \pi^{I} \mathcal{O}_{D}(D)\right),
$$

for each positive integer $i$.

Proof. With 4.4 understood, (1) is easy. The proof of (2) is parallel to that of [27, Proposition 8.2.13]. Let $\mathcal{A}^{p, q}(X)$ and $\mathcal{A}^{p, q}(E)$ be the sheaves of $C^{\infty}$-sections of $\Lambda^{p, q}$ and $\Lambda^{p, q}(E)$. Let

$$
\mathcal{C}^{0}:=\mathcal{A}^{0}(X), \quad \mathcal{C}^{i}:=\mathcal{A}^{0, i}(X) \oplus \mathcal{A}^{0, i-1}(E),(i=1,2), \quad \mathcal{C}^{3}:=\mathcal{A}^{0,2}(E) .
$$

Then the $I$-action makes $\mathcal{C}^{i} I$-equivariant sheaves. The operators $\mathcal{D}_{\mathfrak{p}}^{i}$ in (4.14) define a sequence of $I$-equivariant sheaves:

$$
0 \rightarrow \mathcal{C}^{0} \stackrel{\delta_{\mathfrak{p}}^{0}}{\rightarrow} \mathcal{C}^{1} \stackrel{\delta_{\mathfrak{p}}^{1}}{\rightarrow} \mathcal{C}^{2} \stackrel{\delta_{\mathfrak{p}}^{2}}{\rightarrow} \mathcal{C}^{3} \rightarrow 0 .
$$

This induces the sequence of sheaves over $\hat{X}=X / \iota$ :

$$
0 \rightarrow \pi^{I} \mathcal{C}^{0} \stackrel{\hat{\delta}_{\mathfrak{p}}^{0}}{\rightarrow} \pi^{I} \mathcal{C}^{1} \stackrel{\hat{\delta}_{\mathfrak{p}}^{1}}{\rightarrow} \pi^{I} \mathcal{C}^{2} \stackrel{\hat{\delta}_{\mathfrak{p}}^{2}}{\rightarrow} \pi^{I} \mathcal{C}^{3} \rightarrow 0
$$

Since $\iota$ is free, it can be seen from the $\bar{\partial}$-Poincaré lemma that the sequence (4.17) is exact unless $i \neq 1$. Furthermore, the following map is an isomorphism:

$$
\pi^{I} \mathcal{O}_{X}\left(\mathcal{E}_{\delta}\right) / \phi \pi^{I} \mathcal{O}_{X} \rightarrow \operatorname{ker} \hat{\delta}_{\mathfrak{p}}^{1} / \operatorname{im} \hat{\delta}_{\mathfrak{p}}^{0}, \quad[\lambda] \mapsto(0, \lambda)
$$

For $i>0$,

$$
H^{i}\left(\pi^{I} \mathcal{C}^{1} / \operatorname{im} \hat{\delta}_{\mathfrak{p}}^{0}\right)=0 .
$$

Then we obtain a resolution of $\pi^{I} \mathcal{O}_{X}\left(\mathcal{E}_{\delta}\right) / \phi \pi^{I} \mathcal{O}_{X}=\pi^{I} \mathcal{O}_{D}(D)$ as follows:

$0 \rightarrow \pi^{I} \mathcal{O}_{X}\left(\mathcal{E}_{\delta}\right) / \phi \pi^{I} \mathcal{O}_{X}=\operatorname{ker} \hat{\delta}_{\mathfrak{p}}^{1} / \operatorname{im} \hat{\delta}_{\mathfrak{p}}^{0} \rightarrow \pi^{I} \mathcal{C}^{1} / \operatorname{im} \hat{\delta}_{\mathfrak{p}}^{0} \stackrel{\hat{\delta}_{\mathfrak{p}}^{1}}{\rightarrow} \pi^{I} \mathcal{C}^{2} \stackrel{\hat{\delta}_{\mathfrak{p}}^{2}}{\rightarrow} \pi^{I} \mathcal{C}^{3} \rightarrow 0$.

Since $H^{1}\left(\operatorname{im} \hat{\delta}_{\mathfrak{p}}^{0}\right) \cong H^{1}\left(\pi^{I} \mathcal{C}^{0}\right)$, we have

$$
H^{0}\left(\pi^{I} \mathcal{C}^{1}\right) / \mathcal{D}_{\mathfrak{p}}^{0}\left(H^{0}\left(\pi^{I} \mathcal{C}^{0}\right)\right) \cong H^{0}\left(\pi^{I} \mathcal{C}^{1} / \operatorname{im} \hat{\delta}_{\mathfrak{p}}^{0}\right)
$$

Then we obtain

$$
\begin{aligned}
& H^{0}\left(\pi^{I} \mathcal{O}_{D}(D)\right) \cong \operatorname{ker}\left(H^{0}\left(\pi^{I} \mathcal{C}^{1} / \operatorname{im} \hat{\delta}_{\mathfrak{p}}^{0}\right) \stackrel{\mathcal{D}_{\mathfrak{p}}^{1}}{\rightarrow} H^{0}\left(\pi^{I} \mathcal{C}^{2}\right)=\left(\mathcal{C}^{2}\right)^{I}\right)=H^{1}\left(\left(\mathcal{C}_{\mathfrak{p}}\right)^{I}\right), \\
& H^{i}\left(\pi^{I} \mathcal{O}_{D}(D)\right) \cong \operatorname{ker} \mathcal{D}_{\mathfrak{p}}^{i+1} / \operatorname{im} \mathcal{D}_{\mathfrak{p}}^{i}=H^{i+1}\left(\left(\mathcal{C}_{\mathfrak{p}}\right)^{I}\right) .
\end{aligned}
$$


Corollary 4.18. For $D \in \mathcal{D}(e)^{I}$, if $H^{1}\left(X ; I, \mathcal{O}_{D}(D)\right)=H^{1}\left(\hat{X} ; \pi^{I} \mathcal{O}_{D}(D)\right)=$ 0 , then $\mathcal{D}(e)^{I}$ is smooth at $D$, and the tangent space of $\mathcal{D}(e)^{I}$ at $D$ is identified with $H^{0}\left(X ; I, \mathcal{O}_{D}(D)\right)=H^{0}\left(\hat{X} ; \pi^{I} \mathcal{O}_{D}(D)\right)$.

We call the following the Zariski tangent space of $\mathcal{D}(e)^{I}$ at $D$ :

$$
T_{D}\left(\mathcal{D}(e)^{I}\right):=H^{0}\left(X ; I, \mathcal{O}_{D}(D)\right)=H^{0}\left(\hat{X} ; \pi^{I} \mathcal{O}_{D}(D)\right) .
$$

4.6. Correspondence. Define the map $\tilde{\mathcal{J}}: \mathcal{C}^{*}(E)_{k} \rightarrow \mathcal{P}^{s}(E)_{k}$ by

$$
\tilde{\mathcal{J}}(B, \phi)=\left(\bar{\partial}_{B}, \phi\right) .
$$

Then the restriction of $\tilde{\mathcal{J}}$ to the $I$-invariant part $\mathcal{C}^{*}(E)_{k}^{I}$ induces a submersion

$$
\mathcal{J}^{\prime}:\left(\mathcal{B}_{k}^{*}\right)^{I}=\mathcal{C}^{*}(E)_{k}^{I} / \mathcal{G}_{k+1}^{I} \rightarrow\left(\mathcal{B}_{k}^{s}\right)^{I}:=\mathcal{P}^{s}(E)_{k}^{I} /\left(\mathcal{G}_{k+1}^{\mathbb{C}}\right)^{I} .
$$

The goal of this subsection is the next proposition.

Theorem 4.19. If $\Xi>0$, then the map $\mathcal{J}^{\prime}$ induces a homeomorphism

$$
\mathcal{J}: \mathcal{V}_{t}^{*}(E)^{I} \cong \mathcal{M}^{s}(E)^{I} .
$$

For the proof, we need some preparation. Define $\tilde{\mu}_{t}: \mathcal{C}_{k}^{*} \rightarrow \Omega^{0}(E)_{k-1}$ by the left hand side of the third equation of (4.10) as

$$
\tilde{\mu}_{t}(B, \phi)=i \Lambda_{g} F_{B}+\frac{1}{2}|\phi|^{2}-t .
$$

The restriction of $\tilde{\mu}_{t}$ to the $I$-invariant part $\left(\mathcal{C}_{k}^{*}\right)^{I}$ induces the map $\mu_{t}^{I}:\left(\mathcal{B}_{k}^{*}\right)^{I} \rightarrow$ $\Omega^{0}(E)_{k-1}^{I}$. Let $Z\left(\mu_{t}^{I}\right)=\left(\mu_{t}^{I}\right)^{-1}(0)$. For $v=(B, \phi) \in\left(\mathcal{C}_{k}^{*}\right)^{I}$, by using the Kähler identities $\partial^{*}=i[\Lambda, \bar{\partial}], \bar{\partial}^{*}=-i[\Lambda, \partial]$, we have

$$
\begin{aligned}
T_{[v]} Z\left(\mu_{t}^{I}\right) & =T_{[v]}\left(\mathcal{B}_{k}^{*}\right)^{I} \cap \operatorname{ker} d \tilde{\mu}_{t} \\
& =\left\{(\dot{B}, \dot{\phi}) \mid d^{*} \dot{B}-i \operatorname{Im}(\dot{\phi} \bar{\phi})=0,-i \Lambda_{g} d \dot{B}-\operatorname{Re}(\dot{\phi} \bar{\phi})=0\right\}^{I} \\
& =\left\{(\dot{B}, \dot{\phi}) \mid 2 \bar{\partial}^{*} \dot{B}^{0,1}-\dot{\phi} \bar{\phi}=0\right\}^{I}
\end{aligned}
$$

Note that the last space can be identified with the $L^{2}$-orthogonal complement of the tangent space of the orbit $\mathfrak{p} \cdot\left(\mathcal{G}_{k+1}^{\mathbb{C}}\right)^{I}$ in $T_{\mathfrak{p}}\left(\mathcal{P}_{k}^{s}\right)^{I}$ where $\mathfrak{p}=\tilde{\mathcal{J}}(v) \in\left(\mathcal{P}^{s}\right)^{I}$. From this, we obtain the following:

Proposition 4.20. The map $\left.\mathcal{J}^{\prime}\right|_{Z\left(\mu_{t}^{I}\right)}: Z\left(\mu_{t}^{I}\right) \rightarrow\left(\mathcal{B}_{k}^{s}\right)^{I}$ is a local homeomorphism.

Proof of Theorem 4.19. (Cf. [27, Proposition 8.2.20.) By Proposition 4.20, it suffices to see that $\mathcal{J}$ is bijective. First, we prove $\mathcal{J}$ is surjective. Suppose $(\delta, \phi) \in \mathcal{M}^{s}(E)^{I}$. We have an Hermitian connection $B=A_{h, \delta}$ associated with the holomorphic structure $\delta$. We want to find an $I$-invariant function $\psi \in C^{\infty}(X ; \mathbb{R})^{I}$ such that $\left(A_{h, \delta \cdot f}, \phi\right)$ is a solution to (4.10) for $f=e^{-\psi}$. Note that

$$
A_{h, \delta \cdot f}=A_{h, \delta}-\bar{\partial} \psi+\partial \psi,
$$

and $\left(A_{h, \delta \cdot f}, \phi\right)$ is a $t$-vortex if and only if

$$
i \Lambda_{g} \bar{\partial} \partial \psi+\frac{1}{2} e^{2 \psi}|\phi|^{2}=t-i \Lambda_{g} F_{B}
$$


Since $\phi$ and $\theta:=t-i \Lambda_{g} F_{B}$ are $I$-invariant section and function, they descend on $\hat{X}$, i.e., we find $\hat{\phi}$ and $\hat{\theta}$ such that $\phi=\pi^{*} \hat{\phi}$ and $\theta=\pi^{*} \hat{\theta}$. Consider the following equation for $\hat{\psi} \in C^{\infty}(\hat{X} ; \mathbb{R})$ :

$$
\Delta_{\hat{g}} \hat{\psi}+\frac{1}{2} e^{2 \hat{\psi}}|\hat{\phi}|^{2}=\hat{\theta}
$$

This is a Kazdan-Warner type equation [13], and has a unique solution $\hat{\psi}$ since $\int_{\hat{X}} \hat{\theta} d v^{2} l_{\hat{g}}=\frac{1}{2} \int_{X} \theta d v o l_{g}=\frac{1}{2} \Theta>0$. Then $\psi=\pi^{*} \hat{\psi}$ is an $I$-invariant solution to (4.21).

We prove $\mathcal{J}$ is injective. Suppose $\left(B_{1}, \phi_{1}\right),\left(B_{2}, \phi_{2}\right)$ are $I$-invariant solutions to (4.10) such that $\left(\partial_{B_{1}}, \phi_{1}\right)=\left(\partial_{B_{2}}, \phi_{2}\right) \cdot f$ for some $f \in\left(\mathcal{G}^{\mathbb{C}}\right)^{I}$. By replacing $\left(B_{2}, \phi_{2}\right)$ with $\mathcal{G}^{I}$-equivalent one, if necessary, we may assume $f=e^{-\psi}$ for some $I$-invariant function $\psi$. Since $\left(B_{2}, \phi_{2}\right)$ is an $I$-invariant solution, $\psi$ satisfies (4.21). Since $\left(B_{1}, \phi_{1}\right)$ is an $I$-invariant solution, $\psi=0$ is a solution to (4.21). Moving to the downstairs, we see that the uniqueness of the solution to Kazdan-Warner's equation implies that $\psi=0$.

Recall that $\hat{\mathfrak{s}}_{0} \hat{\otimes} \hat{E}$ is a $\operatorname{Spin}^{c_{-}}$structure on $X \rightarrow \hat{X}$ and $\mathfrak{s}_{0} \otimes E$ is the canonical reduction of $\pi^{*}\left(\hat{\mathfrak{s}}_{0} \hat{\otimes} \hat{E}\right)$. We choose an $I$-invariant closed $(1,1)$ form $\eta$ for the perturbation term of the $I$-invariant Seiberg-Witten equation (4.2).

Corollary 4.22. Let $t=\pi \Lambda_{g} \eta-s_{g} / 2, e=\tilde{c}_{1}(\hat{E})$ and $k=\tilde{c}_{1}(\hat{K})$.

(1) If $\Theta>0$, then

$$
\mathcal{M}\left(\hat{X}, \hat{\mathfrak{s}}_{0} \hat{\otimes} \hat{E}\right) \cong \mathcal{M}\left(X, \mathfrak{s}_{0} \otimes E\right)^{I} \cong \mathcal{V}_{t}^{*}(E)^{I} \cong \mathcal{M}^{s}(E)^{I} \cong \mathcal{D}(e)^{I} .
$$

(2) If $\Theta<0$, then

$$
\mathcal{M}\left(\hat{X}, \hat{\mathfrak{s}}_{0} \hat{\otimes} \hat{E}\right) \cong \mathcal{M}\left(X, \mathfrak{s}_{0} \otimes E\right)^{I} \cong \mathcal{V}_{-t}^{*}\left(E^{-1} \otimes K\right)^{I} \cong \mathcal{M}^{s}\left(E^{-1} \otimes K\right)^{I} \cong \mathcal{D}(k-e)^{I} .
$$

4.7. Witten's perturbation. In the previous subsection, we consider the perturbation by an $I$-invariant $(1,1)$-form $\eta$, and the $\mathrm{Pin}^{-}(2)$-monopole moduli space is identified with the $I$-invariant moduli space of vortices and holomorphic simple pairs. In this subsection, we consider the perturbation as in Theorem 4.6.

Let $(X, \omega, \iota)$ be a compact Kähler surface with anti-holomorphic involution $\iota$, and $E$ and $E^{\prime}$ two $C^{\infty}$ complex line bundles such that $\iota^{*} E \cong \bar{E}$ and $\iota^{*} E^{\prime} \cong \bar{E}^{\prime}$. We suppose $I=\overline{\iota^{*}(\cdot)}$ defines involutions on $E$ and $E^{\prime}$. Consider $\mathcal{P}^{s}(E) \times \mathcal{P}^{s}\left(E^{\prime}\right)$. Let $\mathcal{G}^{\mathbb{C}}=C^{\infty}\left(X, \mathbb{C}^{*}\right)$ act on $\mathcal{P}^{s}(E) \times \mathcal{P}^{s}\left(E^{\prime}\right)$ by

$$
\left(\mathfrak{p}, \mathfrak{p}^{\prime}\right) \cdot f:=\left(\mathfrak{p} \cdot f, \mathfrak{p}^{\prime} \cdot f^{-1}\right) \text { for }\left(\mathfrak{p}, \mathfrak{p}^{\prime}\right) \in \mathcal{P}^{s}(E) \times \mathcal{P}^{s}\left(E^{\prime}\right), f \in \mathcal{G}^{\mathbb{C}} .
$$

Fix a holomorphic structure $\mathcal{N}$ on $N=E \otimes E^{\prime}$, and let $\delta_{\mathcal{N}}$ be the corresponding integrable semiconnection. (Later we assume $\mathcal{N}$, and therefore $\delta_{\mathcal{N}}$, are $I$-invariant.) Let

$$
\mathcal{H}^{s}(E) \times_{\mathcal{N}} \mathcal{H}^{s}\left(E^{\prime}\right):=\left\{\left((\delta, \phi),\left(\delta^{\prime}, \phi^{\prime}\right)\right) \in \mathcal{H}^{s}(E) \times_{\mathcal{N}} \mathcal{H}^{s}\left(E^{\prime}\right) \mid \delta \otimes \delta^{\prime}=\delta_{\mathcal{N}}\right\} .
$$

A natural map $\mathcal{T}: \mathcal{P}^{s}(E) \times \mathcal{P}^{s}\left(E^{\prime}\right) \rightarrow \mathcal{P}^{s}(N)$ given by $\left((\delta, \phi),\left(\delta^{\prime}, \phi^{\prime}\right)\right) \mapsto$ $\left(\delta \otimes \delta^{\prime}, \phi \otimes \phi^{\prime}\right)$ is $\mathcal{G}^{\mathbb{C}}$-invariant. We have a $\mathcal{G}^{\mathbb{C}}$-equivariant commutative 
diagram

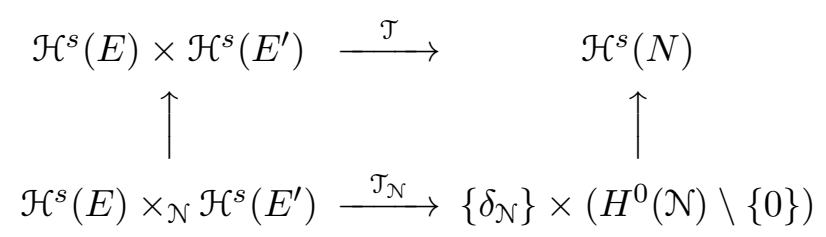

Now suppose $\mathcal{N}$ is $I$-invariant and $\left(H^{0}(\mathcal{N}) \backslash\{0\}\right)^{I} \neq \emptyset$. Choose an $I$-invariant holomorphic section $\xi \in\left(H^{0}(\mathcal{N}) \backslash\{0\}\right)^{I}$. Let

$$
\mathcal{M}^{s}\left(E, E^{\prime}, \mathcal{N}, \xi\right)^{I}=\left(\mathcal{T}_{\mathcal{N}}^{-1}(\xi)\right)^{I} / \mathcal{G}_{\mathbb{C}}^{I}
$$

For $e=c_{1}(E)$ and $e^{\prime}=c_{1}\left(E^{\prime}\right)$, consider the map defined by sum of divisors

$$
\theta: \mathcal{D}(e) \times \mathcal{D}\left(e^{\prime}\right) \rightarrow \mathcal{D}\left(e+e^{\prime}\right)
$$

For $\Delta \in \mathcal{D}\left(e+e^{\prime}\right)$, let

$$
\mathcal{D}_{b}(\Delta):=\theta^{-1}(\Delta)
$$

Then, for $\Delta=Z(\xi)$, we have a natural identification

$$
\mathcal{M}^{s}\left(E, E^{\prime}, \mathcal{N}, \xi\right)^{I} \cong \mathcal{D}_{b}(\Delta)^{I} .
$$

The Zariski tangent space $T_{\left(D, D^{\prime}\right)}\left(\mathcal{D}_{b}(\Delta)^{I}\right)$ of $\mathcal{D}_{b}(\Delta)^{I}$ at $\left(D, D^{\prime}\right)$ is given by

$T_{\left(D, D^{\prime}\right)}\left(\mathcal{D}_{b}(\Delta)^{I}\right)=\operatorname{ker}\left(\theta_{*}: T_{D}\left(\mathcal{D}(e)^{I}\right) \oplus T_{D^{\prime}}\left(\mathcal{D}\left(e^{\prime}\right)^{I}\right) \rightarrow T_{\Delta}\left(\mathcal{D}\left(e+e^{\prime}\right)^{I}\right)\right)$.

For $I$-invariant $D, D^{\prime}, \Delta=D+D^{\prime}$, the inclusions $\emptyset \subset D \subset \Delta, \emptyset \subset D^{\prime} \subset \Delta$ induce the inclusions

$$
\begin{aligned}
& \pi^{I} \mathcal{O}_{X} \subset \pi^{I} \mathcal{O}_{X}(D) \subset \pi^{I} \mathcal{O}_{X}(\Delta), \pi^{I} \mathcal{O}_{X} \subset \pi^{I} \mathcal{O}_{X}\left(D^{\prime}\right) \subset \pi^{I} \mathcal{O}_{X}(\Delta), \\
& \pi^{I} \mathcal{O}_{D}(D)=\pi^{I}\left(\mathcal{O}_{X}(D) / \mathcal{O}_{X}\right) \hookrightarrow \pi^{I}\left(\mathcal{O}_{X}(\Delta) / \mathcal{O}_{X}\right)=\pi^{I} \mathcal{O}_{\Delta}(\Delta), \\
& \pi^{I} \mathcal{O}_{D^{\prime}}\left(D^{\prime}\right)=\pi^{I}\left(\mathcal{O}_{X}\left(D^{\prime}\right) / \mathcal{O}_{X}\right) \hookrightarrow \pi^{I}\left(\mathcal{O}_{X}(\Delta) / \mathcal{O}_{X}\right)=\pi^{I} \mathcal{O}_{\Delta}(\Delta),
\end{aligned}
$$

and therefore the injective maps

$$
\begin{gathered}
T_{D}\left(\mathcal{D}(e)^{I}\right)=H^{0}\left(\pi^{I} \mathcal{O}_{D}(D)\right) \stackrel{i}{\hookrightarrow} H^{0}\left(\pi^{I} \mathcal{O}_{\Delta}(\Delta)\right)=T_{\Delta}\left(\mathcal{D}\left(e+e^{\prime}\right)^{I}\right), \\
T_{D}\left(\mathcal{D}\left(e^{\prime}\right)^{I}\right)=H^{0}\left(\pi^{I} \mathcal{O}_{D^{\prime}}\left(D^{\prime}\right)\right) \stackrel{i^{\prime}}{\hookrightarrow} H^{0}\left(\pi^{I} \mathcal{O}_{\Delta}(\Delta)\right)=T_{\Delta}\left(\mathcal{D}\left(e+e^{\prime}\right)^{I}\right) .
\end{gathered}
$$

Then it can be seen that

$$
\theta_{*}\left(a, a^{\prime}\right)=i(a)+i^{\prime}\left(a^{\prime}\right) .
$$

Proposition 4.23. Let $D_{0}$ be the maximal effective divisor such that $D_{0} \leq$ $D$ and $D_{0} \leq D^{\prime}$. (Note that $D_{0}$ is also I-invariant.) Then there exists an isomorphism $T_{\left(D, D^{\prime}\right)}\left(\mathcal{D}_{b}(\Delta)^{I}\right) \cong H^{0}\left(\pi^{I} \mathcal{O}_{D_{0}}\left(D_{0}\right)\right)$. 
Proof. This is proved by considering the $I$-invariant part or applying $\pi^{I}$ to everything in the proof of [27, Lemma 9.3.3]. The commutative diagram

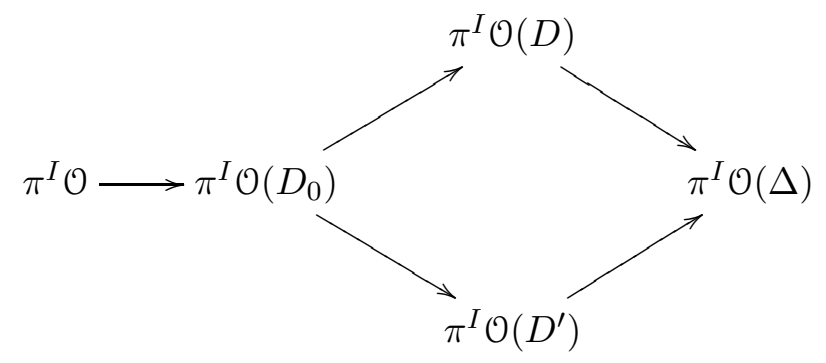

induces a commutative diagram

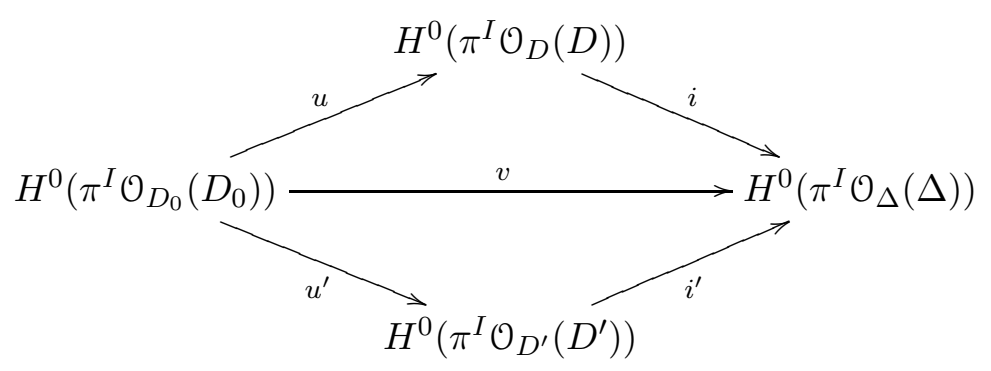

where all of maps are linear monomorphisms. The image of the monomorphism

$$
(-u) \oplus u^{\prime}: H^{0}\left(\pi^{I} \mathcal{O}_{D_{0}}\left(D_{0}\right)\right) \rightarrow H^{0}\left(\pi^{I} \mathcal{O}_{D}(D)\right) \oplus H^{0}\left(\pi^{I} \mathcal{O}_{D^{\prime}}\left(D^{\prime}\right)\right)
$$

is contained in $\operatorname{ker}\left(\theta_{*}\right)$. Therefore

$$
H^{0}\left(\pi^{I} \mathcal{O}_{D_{0}}\left(D_{0}\right)\right) \subset \operatorname{ker}\left(\theta_{*}\right)=T_{\left(D, D^{\prime}\right)}\left(\mathcal{D}_{b}(\Delta)^{I}\right) .
$$

Conversely, let $\left(a, a^{\prime}\right)$ be an element of $\operatorname{ker}\left(\theta_{*}\right)$. Then $i(a)+i^{\prime}\left(a^{\prime}\right)=0$. We have the exact sequences

$$
\begin{gathered}
0 \rightarrow \pi^{I} \mathcal{O}_{D^{\prime}}(-D) \rightarrow \pi^{I} \mathcal{O}_{\Delta} \stackrel{\rho}{\rightarrow} \pi^{I} \mathcal{O}_{D} \rightarrow 0, \\
0 \rightarrow \pi^{I} \mathcal{O}_{D}\left(-D^{\prime}\right) \rightarrow \pi^{I} \mathcal{O}_{\Delta} \stackrel{\rho^{\prime}}{\rightarrow} \pi^{I} \mathcal{O}_{D^{\prime}} \rightarrow 0, \\
0 \rightarrow \pi^{I} \mathcal{O}_{D^{\prime}}\left(D^{\prime}\right) \stackrel{i^{\prime}}{\rightarrow} \pi^{I} \mathcal{O}_{\Delta}(\Delta) \stackrel{r}{\rightarrow} \pi^{I} \mathcal{O}_{D}(\Delta) \rightarrow 0, \\
0 \rightarrow \pi^{I} \mathcal{O}_{D}(D) \stackrel{i}{\rightarrow} \pi^{I} \mathcal{O}_{\Delta}(\Delta) \stackrel{r^{\prime}}{\rightarrow} \pi^{I} \mathcal{O}_{D^{\prime}}(\Delta) \rightarrow 0 .
\end{gathered}
$$

Then $\rho, \rho^{\prime}, r, r^{\prime}$ are restriction maps, since the corresponding maps in the exact sequences without $\pi^{I}$ called the decomposition sequences [1, p.62] are restriction maps. Since $r^{\prime} \circ i=r \circ i^{\prime}=0$ and $i(a)=-i^{\prime}\left(a^{\prime}\right)$, we have

$$
r^{\prime}(i(a))=0, \quad r(i(a))=-r\left(i^{\prime}\left(a^{\prime}\right)\right)=0 .
$$

Hence the restrictions of $i(a) \in H^{0}\left(\pi^{I} \mathcal{O}_{\Delta}(\Delta)\right)$ to $D$ and $D^{\prime}$ are 0 . Let $\tilde{D} \leq \Delta$ be the smallest effective divisor such that $D \leq \tilde{D}, D^{\prime} \leq \tilde{D}$. Then the restriction of $i(a)$ to $\tilde{D}$ is also 0 . By using the decomposition $\Delta=D_{0}+\tilde{D}$, we obtain the exact sequence

$$
0 \rightarrow \pi^{I} \mathcal{O}_{D_{0}}\left(D_{0}\right) \stackrel{v}{\rightarrow} \pi^{I} \mathcal{O}_{\Delta}(\Delta) \stackrel{\tilde{r}}{\rightarrow} \pi^{I} \mathcal{O}_{\tilde{D}}(\Delta) \rightarrow 0 .
$$


Since $\tilde{r}(i(a))=0$, we have an element $b \in H^{0}\left(\pi^{I} \mathcal{O}_{D_{0}}\left(D_{0}\right)\right)$ such that $v(b)=$ $i(a)$. Since $v=i \circ u$ and $i$ is injective, $a=u(b)$. Then $i^{\prime}\left(a^{\prime}+u^{\prime}(b)\right)=$ $-i(a)+\left(i^{\prime} \circ u^{\prime}\right)(b)=-i(a)+v(b)=0$, and hence $a^{\prime}=-u^{\prime}(b)$.

Fix Hermitian metrics $h, h^{\prime}$ on $E$ and $E^{\prime}$, a function $t \in C^{\infty}(X, \mathbb{R})$, an integrable connection $\Sigma$ on $N$ and a nonzero $\bar{\partial}_{\Sigma}$-holomorphic section $\xi \in \Omega^{0}(N) \backslash\{0\}$. (Later we assume that all of them are $I$-invariant.) Let $\mathcal{N}$ be the holomorphic structure on $N$ induced from $\Sigma$. Let $\mathcal{G}=C^{\infty}\left(X, S^{1}\right)$ act on $\left(\mathcal{A}(E, h) \times \Omega^{0}(E)\right) \times\left(\mathcal{A}\left(E^{\prime}, h^{\prime}\right) \times \Omega^{0}\left(E^{\prime}\right)\right)$ by

$$
\left((B, \phi),\left(B^{\prime}, \phi^{\prime}\right)\right) \cdot f=\left((B, \phi) \cdot f,\left(B^{\prime}, \phi^{\prime}\right) \cdot f^{-1}\right) .
$$

Consider the following system of equations:

$$
\left\{\begin{array}{c}
\bar{\partial}_{B} \phi=\bar{\partial}_{B^{\prime}} \phi^{\prime}=0 \\
F_{B}^{0,2}=F_{B^{\prime}}^{0,2}=0 \\
i \Lambda_{g}\left(F_{B}-F_{B^{\prime}}\right)+\frac{1}{2}\left(|\phi|^{2}-\left|\phi^{\prime}\right|^{2}\right)=t \\
B \otimes B^{\prime}=\Sigma \\
\phi \otimes \phi^{\prime}=\xi
\end{array}\right.
$$

Suppose that all of $h, h^{\prime}, t, \Sigma$ and $\eta$ are $I$-invariant. Let

$$
\mathcal{V}_{t}\left(E, E^{\prime}, \Sigma, \xi\right)^{I}=\{I \text {-invariant solutions to (4.24) }\} / \mathcal{G}^{I} \text {. }
$$

Theorem 4.25. The correspondence

$$
\left((B, \phi),\left(B^{\prime}, \phi^{\prime}\right)\right) \mapsto\left(\left(\bar{\partial}_{B}, \phi\right),\left(\bar{\partial}_{B^{\prime}}, \phi^{\prime}\right)\right.
$$

induces a homeomorphism

$$
\mathcal{V}_{t}\left(E, E^{\prime}, \Sigma, \xi\right)^{I} \cong \mathcal{M}^{s}\left(E, E^{\prime}, \mathcal{N}, \xi\right)^{I}
$$

Proof. The proof is similar to that of Theorem 4.19. In this case, we need to find an $I$-invariant function $\psi: X \rightarrow \mathbb{R}$ so that

$$
i \Lambda_{g} \bar{\partial} \partial \psi+\frac{1}{2} e^{2 \psi}|\phi|^{2}-\frac{1}{2} e^{-2 \psi}\left|\phi^{\prime}\right|^{2}=t-i \Lambda_{g}\left(F_{B}-F_{B^{\prime}}\right) .
$$

As before, this equation descends to $\hat{X}$, and it has a unique smooth solution. (See [2] or [19, §3.2].) The rest of the proof is similar.

Corollary 4.26. For $\eta$ as in Theorem 4.6, let $t=\pi \Lambda_{g} \eta^{1,1}-s_{g} / 2$.

$$
\begin{gathered}
\mathcal{M}\left(\hat{X}, \hat{\mathfrak{s}}_{0} \hat{\otimes} \hat{E}\right) \cong \mathcal{M}\left(X ; \mathfrak{s}_{0} \otimes E\right)^{I} \\
\cong \mathcal{V}_{t}\left(E, E^{-1} \otimes K, C^{\vee}, \eta^{2,0}\right)^{I} \cong \mathcal{M}^{s}\left(E, E^{-1} \otimes K, \mathcal{K}, \eta^{2,0}\right)^{I} \cong \mathcal{D}_{b}(\Delta)^{I}
\end{gathered}
$$

\section{Computation and Examples}

The purpose of this section is to compute $\mathrm{Pin}^{-}(2)$-monopole invariants of several concrete examples. 
5.1. Surfaces of general type. In this subsection, we prove Theorem 1.6 on the surfaces of general type and give a series of examples of this type.

Proof of Theorem 1.6. (Cf. [16, Theorem 7.4.1.) The results on the canonical and anti-canonical $\mathrm{Spin}^{c_{-}}$structures follow from Theorem 1.1 and Corollary 1.3 .

Since $X$ is minimal and of general type, $K_{X}^{2}>0$ and $K_{X}$ is numerically effective. The latter condition implies $K_{X} \cdot \omega \geq 0$. But if $K_{X} \cdot \omega=0$, then the Hodge index theorem implies $K_{X}^{2} \leq 0$. Therefore $K_{X} \cdot \omega>0$.

Suppose that $\operatorname{SW}_{X}^{\mathrm{Pin}}(\hat{\mathfrak{s}})$ is nonzero for a $\operatorname{Spin}^{c_{-}}$structure $\hat{\mathfrak{s}}$. Let $\mathfrak{s}$ be the canonical reduction of $\pi^{*} \hat{\mathfrak{s}}$. Let $L$ be the determinant line bundle of $\mathfrak{s}$. Then there exists a complex line bundle $E$ such that $\mathfrak{s}=\mathfrak{s}_{0} \otimes E$. Note that $L=2 E-K_{X}$.

Since $\operatorname{SW}_{X}^{\operatorname{Pin}}(\hat{\mathfrak{s}}) \neq 0, d(\mathfrak{s})=2 d(\hat{\mathfrak{s}}) \geq 0$, and therefore $L^{2} \geq K_{X}^{2}>0$. Then $c_{1}(L)^{+}$is not a torsion class, and this implies that there is no reducible solution and $L \cdot \omega \neq 0$.

Suppose $L \cdot \omega>0$. Since $\operatorname{SW}_{X}^{\operatorname{Pin}}(\hat{\mathfrak{s}}) \neq 0$, there is an $I$-invariant holomorphic structure on $E$ and an $I$-invariant non-zero holomorphic section. Hence $K_{X} \cdot E \geq 0$ because $K_{X}$ is numerically effective. Since $E$ can be written as $E=\left(K_{X}+L\right) / 2, K_{X} \cdot E \geq 0$ implies $K_{X}^{2} \geq-K_{X} \cdot L$. Since $K_{X} \cdot \omega>0$ and $L \cdot \omega>0$, there is $t \geq 0$ such that

$$
\omega \cdot\left(K_{X}+t L\right)=0 .
$$

By the Hodge index theorem, we have

$$
0 \geq\left(K_{X}+t L\right)^{2}=K_{X}^{2}+2 t K_{X} \cdot L+t^{2} L^{2}=: f(t) .
$$

The quadratic function $f(t)$ attains its minimum at $t=-\left(K_{X} \cdot L\right) / L^{2}$ and the minimum is

$$
K_{X}^{2}-\frac{\left(K_{X} \cdot L\right)^{2}}{L^{2}}
$$

Since $L^{2} \geq K_{X}^{2} \geq-K_{X} \cdot L$, this quantity is non-negative, and therefore equal to 0 . Then we have $L^{2}=K_{X}^{2}=-K_{X} \cdot L$, and we see that $f(t) \leq 0$ only when $t=1$. Hence $\left(K_{X}+L\right)^{2}=0$ and $\left(K_{X}+L\right) \cdot \omega=0$. By the Hodge index theorem, we have $K_{X}+L$ is a torsion class, and therefore $E$ is also a torsion class. Since $E$ has an $I$-invariant non-zero holomorphic section, $E$ is an $I$-equivariant trivial bundle. This means $\hat{\mathfrak{s}}$ is the canonical $\mathrm{Spin}^{c_{-}}$ structure.

On the other hand, in the case when $L \cdot \omega>0, \operatorname{SW}_{X}^{\text {Pin }}(\hat{\mathfrak{s}}) \neq 0$ implies the existence of an $I$-invariant holomorphic structure on $E-K_{X}$, and an $I$-invariant holomorphic section on it. Arguing similarly, we can prove that $K_{X}-L$ is a torsion class, and $\hat{\mathfrak{s}}$ is the anti-canonical $\mathrm{Spin}^{c_{-}}$structure.

For a positive integer $k$, let $M_{4 k}$ be the hypersurface in $\mathbb{C P}^{3}$ defined by real polynomials of degree $4 k$, e.g., $\sum_{j=0}^{3} x_{j}^{4 k}$. If $k>1$, then $M_{4 k}$ is a minimal Kähler surfaces of general type. Define the antiholomorophic free involution $\iota$ by

$$
\left[x_{0}, x_{1}, x_{2}, x_{3}\right] \mapsto\left[\bar{x}_{1},-\bar{x}_{0}, \bar{x}_{3},-\bar{x}_{2}\right] .
$$

Let $\hat{M}_{4 k}=M_{4 k} / \iota$. We check the assumptions.

Lemma 5.1. There is a lift of $w_{2}\left(\hat{M}_{4 k}\right)$ in the torsion part of $H^{2}\left(\hat{M}_{4 k} ; \mathbb{Z}\right)$. 
Proof. See [11]. The proof in [11] is on $\hat{M}_{4}$, but it works well for $\hat{M}_{4 k}$.

Proposition 5.2. $w_{2}(\hat{K})=0$ and $w_{2}\left(\hat{M}_{4 k}\right)=w_{1}\left(\ell_{\mathbb{R}}\right)^{2} \cdot \pi^{*}: H^{1}\left(\hat{M}_{4 k} ; \mathbb{Z}_{2}\right) \rightarrow$ $H^{1}\left(M_{4 k} ; \mathbb{Z}_{2}\right)$ is surjective.

Proof. The fact that $w_{2}(\hat{K})=0$ follows from that the canonical bundle $K$ of $M_{4 k}$ is given by

$$
K=(4 k-4) H,
$$

where $H$ is the hyperplane section. By Lemma 5.1] and [17, $\S 1$, Remark $3(2)]$, there exists a class $\alpha \in H^{1}\left(\hat{M}_{4 k} ; \ell\right)$ such that $w_{2}\left(\hat{M}_{4 k}\right)=\alpha \cup \alpha$. Since $\pi_{1}\left(M_{4 k}\right)=1, \pi_{1}\left(\hat{M}_{4 k}\right)=\mathbb{Z} / 2, \alpha$ must be $w_{1}\left(\ell_{\mathbb{R}}\right)$ and $\pi^{*}$ is surjective.

Corollary 5.3. There exists a canonical $\mathrm{Spin}^{c_{-}}$structure $\mathfrak{s}_{0}$ on $M_{4 k} \rightarrow \hat{M}_{4 k}$.

Proposition 5.4. The moduli space for $\left(\hat{M}_{4 k}, \hat{\mathfrak{s}}_{0}\right)$ is orientable and its virtual dimension is 0 . Therefore the $\mathrm{Pin}^{-}(2)$-monopole invariant of $\left(\hat{M}_{4 k}, \hat{\mathfrak{s}}_{0}\right)$ can be defined as a $\mathbb{Z}$-valued invariant.

Proof. Note $b_{1}^{\ell}\left(\hat{M}_{4 k}\right)=0$. In order to prove the orientability of the moduli space, it suffices to prove the Dirac index, ind $D$, of $\hat{\mathfrak{s}}_{0}$ is even by [18, Proposition 2.15]. Let $d\left(\hat{\mathfrak{s}}_{0}\right)$ be the virtual dimension of the moduli space. Since $\iota$ is free, we have $d\left(\hat{\mathfrak{s}}_{0}\right)=\frac{1}{2} d\left(\mathfrak{s}_{0}\right)=0$. Then

$$
0=d\left(\hat{\mathfrak{s}}_{0}\right)=\text { ind } D-\left(b_{0}^{\ell}-b_{1}^{\ell}+b_{+}^{\ell}\right) .
$$

Since $\ell$ is nontrivial, $b_{0}^{\ell}=0$. Therefore

$$
\text { ind } D=b_{+}^{\ell}=\frac{1}{2}\left(1+b_{+}\left(M_{4 k}\right)\right)=\frac{1}{2}\left(\frac{4 k}{3}\left\{16 k^{2}-24 k+11\right\}\right) \text {. }
$$

(For the calculation of $b_{+}\left(M_{4 k}\right)$, see e.g. [15, Example 4.27].) Therefore ind $D$ is even.

Remark 5.5. Note that $\hat{M}_{4}$ is diffeomorphic to an Enriques surface. If $k>1$, then all of the ordinary Seiberg-Witten invariants of $\hat{M}_{4 k}$ are zero by a theorem due to S. Wang [30].

5.2. Elliptic surfaces. In this subsection, we compute the $\mathrm{Pin}^{-}(2)$-monopole invariants of the quotient manifolds of some elliptic surfaces. First, we construct anti-holomorphic involutions on certain elliptic surfaces over $\mathbb{C P}^{1}$.

A method to construct elliptic fibrations by using hyperelliptic involutions is given in Gompf-Stipsicz's book [9], §3.2. Let $\Sigma_{k}$ be a Riemannian surface of genus $k$, and $h_{k}: \Sigma_{k} \rightarrow \Sigma_{k}$ be a hyperelliptic involution. Take the diagonal $\mathbb{Z}_{2}$-action $h_{k} \times h_{1}$ on $\Sigma_{k} \times \Sigma_{1}$. Dividing by the $\mathbb{Z}_{2}$-action $h_{k} \times h_{1}$, we obtain the quotient $\left(\Sigma_{k} \times \Sigma_{1}\right) / \mathbb{Z}_{2}$ with $4(2 k+2)$ singular points. Resolving the singular points produces a complex manifold $X(k+1)$. Dividing the projection $\operatorname{pr}_{1}: \Sigma_{k} \times \Sigma_{1} \rightarrow \Sigma_{k}$ and extending it to the resolution, we obtain the elliptic fibration $\varpi: X(k+1) \rightarrow \mathbb{C P}^{1}$. It is well-known that $X(n)$ is diffeomorphic to $E(n)$, the fiber sum of $E(1)=\mathbb{C P}^{2} \# 9 \overline{\mathbb{C P}}^{2}$.

We construct an anti-holomorphic free involution on $X(2 n)$. Take the antipodal map $\iota_{0}$ on $\mathbb{C P}^{1}=\mathbb{C} \cup\{\infty\}$ defined by $z \mapsto z^{*}:=-1 / \bar{z}$. Choose $k$ distinct points $a_{1}, \ldots, a_{k}$ on $\mathbb{C P}^{1}$ satisfying $0<\left|a_{i}\right|<1$. Let $\Sigma_{k}$ be the hyperelliptic curve defined by the equation

$$
w^{2}=z\left(z-a_{1}\right)\left(z-a_{1}^{*}\right) \cdots\left(z-a_{k}\right)\left(z-a_{k}^{*}\right),
$$


and $\Sigma_{k} \rightarrow \mathbb{C P}^{1}$ the associated double covering branched at $a_{1}, a_{1}^{*}, \ldots, a_{k}$, $a_{k}^{*}, 0, \infty$. Then the antipodal map $\iota_{0}$ on the base $\mathbb{C P}^{1}$ can be lifted to an anti-holomorphic map $\sigma_{k}$ on $\Sigma_{k}$ with order 2 if $k$ is odd, and with order 4 if $k$ is even.

Suppose $k=2 n-1$ for a positive integer $n$. Take the diagonal action $\sigma_{2 n-1} \times \sigma_{1}: \Sigma_{2 n-1} \times \Sigma_{1} \rightarrow \Sigma_{2 n-1} \times \Sigma_{1}$. Then $\sigma_{2 n-1} \times \sigma_{1}$ descends to a free involution on the quotient $\left(\Sigma_{2 n-1} \times \Sigma_{1}\right) / \mathbb{Z}_{2}$. Furthermore we can easily extend it to an anti-holomorphic free involution $\iota$ on $X(2 n)$ which covers the antipodal map $\iota_{0}$ on the base $\mathbb{C P}^{1}$.

Proposition 5.6. The surface $X(2 n)$ admits a Kähler form $\omega$ such that $\iota^{*} \omega=-\omega$.

Proof. We can easily construct a Kähler form $\omega_{0}$ on $\Sigma_{k} \times \Sigma_{1}$ such that $\left(h_{k} \times h_{1}\right)^{*} \omega_{0}=\omega_{0}$ and $\left(\sigma_{k} \times \sigma_{1}\right)^{*} \omega_{0}=-\omega_{0}$. Then $\omega_{0}$ induces a singular Kähler form $\hat{\omega}_{0}$ on $\left(\Sigma_{k} \times \Sigma_{1}\right) / \mathbb{Z}_{2}$. By the results due to Fujiki [8], we can obtain a Kähler form $\omega$ on $X(2 n)$. Moreover we can choose $\omega$ such that $\iota^{*} \omega=-\omega$.

Let $\hat{X}(2 n)=X(2 n) / \iota$. By construction, $\varpi: X(2 n) \rightarrow \mathbb{C P}^{1}$ descends to $\hat{\varpi}: \hat{X}(2 n) \rightarrow \mathbb{R P}^{2}$. The general fiber of $\hat{\varpi}$ is a torus. Note that $X(2)=E(2)$ is a $K 3$ surface.

Proposition 5.7. The quotient manifold $\hat{X}(2)=X(2) / \iota$ is diffeomorphic to an Enriques surface.

Proof. ([5] and [4, §15.1.) Take an $I$-invariant holomorphic form $\phi$ on $X(2)$. By the Calabi-Yau theorem, there exists a unique Kähler-Einstein metric. Then $\phi$ and $\omega$ induce a hyper-Kähler structure on $X(2)$. There exists a complex structure for which $\iota$ is a holomorphic free involution. Thus $\hat{X}(2)$ is an Enriques surface.

Since $X(2 n)$ is diffeomorphic to the fiber sum of $E(2)=K 3$ with $E(2 n-$ 2), $\hat{X}(2 n)$ is diffeomorphic to the fiber sum of the fibration $\hat{\varpi}: \hat{X}(2) \rightarrow \mathbb{R} \mathrm{P}^{2}$ with $E(n-1)$.

Proposition 5.8. If $k \equiv 2$ modulo 4 , then there exists a canonical Spin ${ }^{c_{-}}$ structure $\hat{\mathfrak{s}}_{0}$ on $X(k) \rightarrow \hat{X}(k)$.

Proof. Since $\hat{X}(4 m+2)$ is the fiber sum of $\hat{\varpi}: \hat{X}(2) \rightarrow \mathbb{R} \mathrm{P}^{2}$ and $E(2 m)$, it is easy to see that $\hat{X}(4 m+2)$ is a non-spin manifold with $\pi_{1}(\hat{X}(4 m+2))=\mathbb{Z} / 2$ whose intersection form is isomorphic to

$$
(2 m+1)\left(-E_{8}\right) \oplus(4 m+1) H,
$$

where $H$ is a hyperbolic form. Then it follows from a result of HambletonKreck [12] (Cf. [29]) that $\hat{X}(4 m+2)$ is homeomorphic to the connected sum

$$
\Sigma \#(2 m+1)\left|E_{8}\right| \#(4 m+1)\left(S^{2} \times S^{2}\right),
$$

where $\Sigma$ is a rational homology 4 -sphere such that $\pi_{1}(\Sigma)=\mathbb{Z} / 2$ and $w_{2}(\Sigma) \neq$ 0 , and $\left|E_{8}\right|$ is the $E_{8}$-manifold, i.e., the simply-connected topological manifold whose intersection form is isomorphic to $-E_{8}$. Since $\left|E_{8}\right|$ and $S^{2} \times S^{2}$ are spin and $w_{2}(\Sigma)$ is a torsion class, $w_{2}(\hat{X}(4 m+2))$ is a torsion class. 
Since the canonical divisor $K$ of $X(n)$ is $K=(n-2) F$, where $F$ is a general fiber, we can see that $\tilde{c}_{1}(\hat{K})$ is divided by 2 if $n=4 m+2$. The rest of the proof is similar to that of Proposition 5.2.

Take an $I$-invariant divisor $D_{k}$ of $X(n)$ of the form

$$
D_{k}=\sum_{i=1}^{k}\left(F_{i}+I F_{i}\right),
$$

where $F_{i}$ are general fibers. Let $E_{k}$ be the line bundle associated to $D_{k}$. Then $E_{k}$ can be written as the pull-back $E_{k}=\varpi^{*} L$ where $\varpi: X(n) \rightarrow \mathbb{C P}^{1}$ is the elliptic fibration and $L$ is a line bundle over $\mathbb{C P}^{1}$ of degree $2 k$. Let $\hat{E}_{k}=E_{k} / I$.

The next is an analogue of [7, Proposition 4.2] or [3, Proposition 42].

Theorem 5.9. Let $\hat{X}=\hat{X}(4 m+2)$ and $\hat{\mathfrak{s}}_{k}=\hat{\mathfrak{s}}_{0} \hat{\otimes} \hat{E}_{k}$. The moduli space $\hat{\mathcal{M}}\left(\hat{X}, \hat{\mathfrak{s}}_{k}\right)$ is orientable and the corresponding invariant is

$$
\mathrm{SW}^{\mathrm{Pin}}\left(\hat{X}, \hat{\mathfrak{s}}_{k}\right)= \pm\left(\begin{array}{c}
2 m \\
k
\end{array}\right)
$$

The rest of this section is devoted to the proof of Theorem [5.9, For $q \in \mathbb{C P}^{1}$, let $q^{*}=\iota_{0}(q)$ where $\iota_{0}$ is the antipodal map. Choose $2 m$ distinct points $q_{1}, \ldots, q_{2 m}$ on $\mathbb{C P}^{1}$ such that all of $q_{1}, \ldots, q_{2 m}$ and $q_{1}^{*}, \ldots, q_{2 m}^{*}$ are distinct. Let $F_{i}=\varpi^{-1}\left(q_{i}\right)$. Then $I F_{i}=\varpi^{-1}\left(q_{i}^{*}\right)$, and we obtain an $I$ invariant canonical divisor $D_{m}=\sum_{i=1}^{2 m}\left(F_{i}+I F_{i}\right)$ of $X=X(4 m+2)$. Let $\eta$ be the corresponding $I$-invariant holomorphic section on the canonical bundle $\mathcal{K}$. By Corollary 4.26, the $\operatorname{Pin}^{-}(2)$-monopole moduli space $\mathcal{M}\left(\hat{X}, \hat{\mathfrak{s}}_{k}\right)$ is identified with

$$
\mathcal{V}_{t}\left(E, E^{\prime}, \Sigma, \eta\right)^{I} \cong \mathcal{M}^{s}\left(E, E^{\prime}, \mathcal{K}, \eta\right)^{I} \cong \mathcal{D}_{b}(\Delta)^{I},
$$

where $E^{\prime}=K \otimes E^{-1}$ and $\Delta=Z(\eta)$.

Lemma 5.10. The moduli space $\mathcal{M}\left(\hat{X}, \hat{\mathfrak{s}}_{k}\right)$ is 0 -dimensional and orientable.

Proof. Let $\mathfrak{s}_{k}$ be the $\operatorname{Spin}^{c}$ structure on $X$ of the canonical reduction of $\pi^{*} \hat{\mathfrak{s}}_{k}$. Note that $c_{1}(L)^{2}=0, \tau(X)=-16(2 m+1)$ and $2 e(X)+3 \tau(X)=0$, where $L=K^{-1} \otimes E_{k}^{2}$ is the determinant line bundle, and $e(X)$ and $\tau(X)$ are the Euler characteristic and signature of $X$. Then the virtual dimension $d\left(\mathfrak{s}_{k}\right)$ of the Seiberg-Witten moduli space of $\left(X, \mathfrak{s}_{k}\right)$ is

$$
d\left(\mathfrak{s}_{k}\right)=\frac{1}{4}\left(c_{1}(L)^{2}-2 e(X)-3 \tau(X)\right)=0 .
$$

Then we have $d\left(\hat{\mathfrak{s}_{k}}\right)=d\left(\mathfrak{s}_{k}\right) / 2=0$.

Since the index of the Dirac operator $D_{\hat{A}}$ on $\hat{\mathfrak{s}}_{k}$ is a half of that on $\mathfrak{s}$, we have

$$
\text { ind } D_{\hat{A}}=\frac{1}{2}\left(\frac{1}{4}\left(c_{1}(L)^{2}-\tau(X)\right)\right)=4 m+2 .
$$

Especially, ind $D_{\hat{A}}$ is even. Then the moduli space is orientable by [18, Proposition 2.15].

Proposition 5.11. Every $\mathrm{Pin}^{-}(2)$-monopole solution corresponding to a divisor $D \in \mathcal{D}_{b}(\Delta)^{I}$ is non-degenerate. 
Proof. Since $D_{m}-D$ and $D$ have no intersection for $D \in \mathcal{D}_{b}(\Delta)^{I}$, Proposition 4.23 implies that the first cohomology $H^{1}$ of the deformation complex of the solution corresponding to $D$ is 0 . Therefore the second cohomology $H^{2}$ is also 0 since $d\left(\hat{\mathfrak{s}_{k}}\right)=0$ and $H^{1}=0$.

For a subset $\left\{i_{1}, \ldots, i_{k}\right\} \subset\{1, \ldots, 2 m\}$, we have an $I$-invariant divisor $\sum_{j=1}^{k}\left(F_{i_{j}}+I F_{i_{j}}\right) \in \mathcal{D}_{b}(\Delta)^{I}$. Since this correspondence is bijective, the number of elements in $\mathcal{D}_{b}(\Delta)^{I}$ is $\left(\begin{array}{c}2 m \\ k\end{array}\right)$. In order to complete the proof of Theorem [5.9, we show that all of the divisors in $\mathcal{D}_{b}(\Delta)^{I}$ have same orientation.

Proposition 5.12. For distinct points $b_{1}, \ldots, b_{k} \in \mathbb{C P}^{1}$, consider the divisor $B=b_{1}+\cdots+b_{k}$. Let $F_{j}=\varpi^{*}\left(b_{j}\right)$ and $D=F_{1}+\cdots+F_{j}$. Then $\varpi^{*}: H^{0}\left(\mathcal{O}_{\mathbb{C P}^{1}}(B)\right) \rightarrow H^{0}\left(\mathcal{O}_{X}(D)\right)$ is an isomorphism, and

$$
H^{0}\left(\mathcal{O}_{X}(D)\right)=\mathbb{C}^{k+1}, \quad H^{1}\left(\mathcal{O}_{X}(D)\right)=0, \quad H^{2}\left(\mathcal{O}_{X}(D)\right)=\mathbb{C}^{4 m-k}
$$

Proof. Consider the short exact sequence

$$
0 \rightarrow \mathcal{O}_{X} \rightarrow \mathcal{O}_{X}(D) \rightarrow \mathcal{O}_{D}(D) \rightarrow 0
$$

and its associated long exact sequence. Note that $\mathcal{O}_{F_{j}}(D)$ is the holomorphic normal bundle of $F_{j} \subset X$. Since this is holomorphicallly trivial and the genus of $F_{j}$ is 1 , we have

$$
H^{0}\left(\mathcal{O}_{D}(D)\right)=H^{1}\left(\mathcal{O}_{D}(D)\right)=\mathbb{C}^{k} .
$$

Since $X$ is simply-connected, $H^{1}\left(\mathcal{O}_{X}\right)=0$ and therefore

$$
H^{1}\left(\mathcal{O}_{X}(D)\right)=H^{0}\left(\mathcal{O}_{X}\right) \oplus H^{1}\left(\mathcal{O}_{D}(D)\right)=\mathbb{C}^{k+1} .
$$

By the Serre duality,

$$
H^{2}\left(\mathcal{O}_{X}(D)\right)=H^{0}\left(\mathcal{O}_{X}(K-D)\right)=\mathbb{C}^{4 m-k} .
$$

Also we have $H^{1}\left(\mathcal{O}_{X}(D)\right)=0$.

Let $\mathfrak{t}=(B, \phi, \psi) \in \mathcal{A}(E, h) \times \Omega^{0,0}(E) \times \Omega^{2,0}\left(E^{-1}\right)$ be an $I$-invariant solution to (4.24), i.e., $\left((B, \phi),\left(B^{\prime}, \phi^{\prime}\right)\right)$ where $B^{\prime}=\Sigma \otimes B^{-1}$ and $\phi^{\prime}=\psi$ is $I$-invariant and satisfies (4.24). The deformation complex at $\mathfrak{t}$ is given by

$$
\begin{aligned}
& \left(i \Omega_{X}^{0}\right)^{I} \stackrel{D_{+}^{0}}{\rightarrow}\left(i \Omega_{X}^{1} \oplus \Omega^{0,0}(E) \oplus \Omega^{2,0}\left(E^{-1}\right)\right)^{I} \stackrel{D_{\ddagger}^{1}}{\rightarrow}\left(\Omega^{0,1}(E) \oplus i\left(\Omega_{X}^{0,2} \oplus \Omega_{X}^{1,1}\right)\right)^{I}, \\
& D_{\mathfrak{t}}^{0}(f)=\left(\begin{array}{c}
d f \\
f \phi \\
-f \psi
\end{array}\right), \quad D_{\mathfrak{t}}^{1}(\dot{B}, \dot{\phi}, \dot{\psi})=\left(\begin{array}{c}
\dot{B}^{0,1} \phi+w\left(\dot{B}^{0,1}\right)^{*} \bar{\psi}+\bar{\partial}_{B} \dot{\phi}+\bar{\partial}_{B}^{*} \overline{\dot{\psi}} \\
\bar{\partial} \dot{B}^{0,1}-\frac{1}{2} \bar{\psi} \overline{\dot{\phi}}-\frac{1}{2} \dot{\bar{\psi}} \bar{\phi} \\
\left.\Lambda_{g} d \dot{B}-i[\operatorname{Re}(\dot{\phi} \bar{\phi})-\operatorname{Re}(\bar{\psi} \dot{\psi})]\right\} \omega
\end{array}\right),
\end{aligned}
$$

where $w\left(\dot{B}^{0,1}\right)^{*}$ is the adjoint of the multiplication operator $\dot{B}^{0,1} \wedge \cdot$ The adjoint of $D_{\mathrm{t}}^{0}$ is

$$
\left(D_{\mathfrak{t}}^{0}\right)^{*}(\dot{B}, \dot{\phi}, \dot{\psi})=d^{*} \dot{B}-i \operatorname{Im}(\dot{\phi} \bar{\phi})+i \operatorname{Im}(\bar{\psi} \dot{\psi}) .
$$


Set $\dot{b}=\sqrt{2} \dot{B}, U=\frac{1}{\sqrt{2}} \phi, V=\frac{1}{\sqrt{2}} \psi$. Replace $\dot{b}$ by $\dot{b}^{0,1}$ via the identification $i \Omega_{X} \cong \Omega^{0,1}$. We introduce the operators $Q_{\mathfrak{t}}$ and $Q_{\mathfrak{t}}^{0}$ by

$$
\begin{aligned}
& Q_{\mathfrak{t}}=\left(\begin{array}{cccc}
-\mathrm{id} & 0 & 0 & 0 \\
0 & \sqrt{2} \text { id } & 0 & 0 \\
0 & 0 & \frac{1}{\sqrt{2} i \omega} & 0 \\
0 & 0 & 0 & \frac{1}{\sqrt{2}}
\end{array}\right)\left(\begin{array}{c}
D_{\mathrm{t}}^{1} \\
\left(D_{\mathrm{t}}^{0}\right)^{*}
\end{array}\right), \\
& Q_{\mathfrak{t}}\left(\begin{array}{c}
\dot{b} \\
\dot{\phi} \\
\dot{\psi}
\end{array}\right)=\left(\begin{array}{c}
-b^{0,1} U-w\left(\dot{b}^{0,1}\right)^{*} \bar{V}-\bar{\partial}_{B} \dot{\phi}-\bar{\partial}_{B}^{*} \overline{\dot{\psi}} \\
\bar{\partial} \dot{b}^{0,1}-\bar{V} \overline{\dot{\phi}}-\overline{\dot{\psi}} \bar{U} \\
\bar{\partial}^{*} \dot{b}^{0,1}-\dot{\phi} \bar{U}+\bar{V} \dot{\psi}
\end{array}\right), \\
& Q_{\mathfrak{t}}^{0}\left(\begin{array}{c}
\dot{b} \\
\dot{\phi} \\
\dot{\psi}
\end{array}\right)=\left(\begin{array}{c}
-\bar{\partial}_{B} \dot{\phi}-\bar{\partial}_{B}^{*} \overline{\dot{\psi}} \\
\bar{\partial} \dot{b}^{0,1} \\
\bar{\partial}^{*} \dot{b}^{0,1}
\end{array}\right) .
\end{aligned}
$$

Let $K_{0}$ and $C_{0}$ be the kernel and cokernel of $Q_{\mathrm{t}}^{0}$, respectively,

$$
\begin{aligned}
K_{0} & :=\operatorname{ker} Q_{\mathfrak{t}}^{0}=\operatorname{ker}\left(\bar{\partial}_{B}+\bar{\partial}_{B}^{*}\right)^{I} \oplus \mathbb{H}^{1}\left(\mathcal{O}_{X}\right)^{I}, \\
C_{0} & :=\operatorname{coker} Q_{\mathfrak{t}}^{0}=\operatorname{ker}\left(\bar{\partial}_{B}^{*} \oplus \bar{\partial}_{B}\right)^{I} \oplus \mathbb{H}^{2}\left(\mathcal{O}_{X}\right)^{I} \oplus \mathbb{H}^{0}(\mathcal{O})^{I} .
\end{aligned}
$$

Note that $\mathbb{H}^{1}\left(\mathcal{O}_{X}\right)=0$ since $b_{1}(X)=0$. By Proposition 5.12, $\operatorname{ker}\left(\bar{\partial}_{B}^{*} \oplus \bar{\partial}_{B}\right)=$ $H^{1}\left(\mathcal{O}_{X}(D)\right)=0$.

To see the orientation of the solution $\mathfrak{t}$, we consider

$$
\operatorname{pr}_{C_{0}} \circ\left(\left.Q_{\mathfrak{t}}\right|_{K_{0}}\right): K_{0}=\operatorname{ker}\left(\bar{\partial}_{B}+\bar{\partial}_{B}^{*}\right)^{I} \rightarrow C_{0}=H^{2}\left(\mathcal{O}_{X}\right)^{I} \oplus H^{0}\left(\mathcal{O}_{X}\right)^{I} .
$$

This can be identified with

$$
\begin{gathered}
R_{\mathrm{t}}: H^{0}(D)^{I} \oplus H^{0}(K-D)^{I} \rightarrow H^{0}(K)^{I} \oplus H^{0}\left(\mathcal{O}_{X}\right)^{I}, \\
R_{\mathfrak{t}}=\left(\begin{array}{c}
\dot{\phi} \\
\dot{\psi}
\end{array}\right)=\left(\begin{array}{c}
-V \dot{\phi}-U \dot{\psi} \\
\int(-\langle\dot{\phi}, U\rangle+\langle\dot{\psi}, V\rangle) d \mathrm{vol}_{g}
\end{array}\right) .
\end{gathered}
$$

Note that $R_{\mathrm{t}}$ is a linear isomorphism. If we fix orientations of the domain and target of $R_{\mathrm{t}}$, the orientation of the solution $\mathfrak{t}$ is determined by the sign of the determinant of $R_{\mathrm{t}}$. (See e.g. [27, [19].) We want to represent $R_{\mathrm{t}}$ by a matrix with some explicit bases of $H^{0}(D)^{I} \oplus H^{0}(K-D)^{I}$ and $H^{0}(K)^{I} \oplus H^{0}\left(\mathcal{O}_{X}\right)^{I}$.

Consider a complex manifold $Z$ with a divisor $D$. Let $\mathcal{L}_{D}$ be the holomorphic line bundle associated with $D$. Then $H^{0}\left(\mathcal{L}_{D}\right)$ can be identified with the space of meromorphic functions $f$ such that $\lambda f$ are holomorphic for every local defining function $\lambda$ of $D$. This space is denoted by $\mathfrak{M}(D)$. Note that $1 \in \mathfrak{M}(D)$ corresponds to the holomorphic section $\phi_{D}$ defined by the divisor $D$.

Suppose $\iota$ is an anti-holomorphic free involution on $Z$ and the divisor $D$ is $I$-invariant. The $I$-action on $H^{0}\left(\mathcal{L}_{D}\right)=\mathfrak{M}(D)$ is given by $f \mapsto \overline{\iota^{*} f}$.

Consider $(Z, \iota)=\left(\mathbb{C P}^{1}, \iota_{0}\right)$ where $\iota_{0}$ is the antipodal map. Choose $p \in$ $\mathbb{C} \subset \mathbb{C P}^{1}$ such that $|p|=1$. Let $p^{*}=\iota_{0}(p)=-1 / \bar{p}=-p$. Take the $\iota_{0^{-}}$ invariant divisor $B=p+p^{*}$. In terms of meromorphic functions, we can take the following for a complex basis of $\mathfrak{M}(B)=H^{0}\left(\mathcal{L}_{B}\right)$.

$$
1, \frac{z-p^{*}}{z-p}, \frac{z-p}{z-p^{*}} \text {. }
$$


We want to have a (real) basis for $\mathfrak{M}(B)^{I}=H^{0}\left(\mathcal{L}_{B}\right)^{I}$. Via the projection to the $I$-invariant part, $f \mapsto \frac{1}{2}(f+I f)$, we see that the following is a real basis for $\mathfrak{M}(B)^{I}=H^{0}\left(\mathcal{L}_{B}\right)^{I}$ :

$$
1, \quad i \frac{z^{2}+p^{2}}{z^{2}-p^{2}}, \quad \frac{2 p z}{z^{2}-p^{2}} .
$$

Let us consider the case of $\varpi: X=X(4 m+2) \rightarrow \mathbb{C P}^{1}$ with the antiholomorphic involution $\iota$. Choose $2 m$ distinct points $p_{1}, \ldots, p_{k}, q_{1}, \ldots, q_{2 m-k}$ on $\mathbb{C P}^{1}$ such that $\left|p_{j}\right|=1,\left|q_{l}\right|=1$, and all of $p_{j}, p_{j}^{*}, q_{l}, q_{l}^{*}$ are distinct. Take the following divisors on $\mathbb{C P}^{1}$ :

$$
B_{D}=\sum_{j=1}^{k}\left(p_{j}+p_{j}^{*}\right), \quad B_{K-D}=\sum_{l=1}^{2 m-k}\left(q_{l}+q_{l}^{*}\right), \quad B_{K}=B_{D}+B_{K-D} .
$$

Then $K=\varpi^{*} B_{K}$ is a canonical divisor of $X$. Let $D=\varpi^{*} B_{D}$. Then $K-D=\varpi^{*} B_{K-D}$. Let

$$
P_{j}^{1}=i \frac{z^{2}+p_{j}^{2}}{z^{2}-p_{j}^{2}}, \quad P_{j}^{2}=\frac{2 p_{j} z}{z^{2}-p_{j}^{2}}, \quad Q_{l}^{1}=i \frac{z^{2}+q_{l}^{2}}{z^{2}-q_{l}^{2}}, \quad Q_{l}^{2}=\frac{2 q_{l} z}{z^{2}-q_{l}^{2}} .
$$

Then $\left\{1, P_{j}^{1}, P_{j}^{2}\right\}(j=1, \ldots, k)$ is a basis of $\mathfrak{M}\left(B_{D}\right)^{I}=H^{0}\left(B_{D}\right)^{I} \cong H^{0}(D)^{I}$. Similarly, $\left\{1, Q_{l}^{1}, Q_{l}^{2}\right\}(l=1, \ldots, 2 m-k)$ and $\left\{1, P_{j}^{1}, P_{j}^{2}, Q_{l}^{1}, Q_{l}^{2}\right\}(j=$ $1, \ldots, k, l=1, \ldots, 2 m-k)$ are bases of $\mathfrak{M}\left(B_{K-D}\right)^{I}=H^{0}\left(B_{K-D}\right)^{I} \cong$ $H^{0}(K-D)^{I}$ and $\mathfrak{M}\left(B_{K}\right)=H^{0}\left(B_{K}\right)^{I} \cong H^{0}(K)^{I}$, respectively.

Now the divisor $D$ corresponds to an $I$-invariant solution $\mathfrak{t}=(B, \phi, \psi)$ such that $\phi^{-1}(0)=D$ and $\psi^{-1}(0)=K-D$. We may assume the following correspondence,

$$
\begin{gathered}
1 \in \mathfrak{M}\left(B_{D}\right)^{I} \longleftrightarrow U=\frac{1}{\sqrt{2}} \phi \in H^{0}(D)^{I} \\
1 \in \mathfrak{M}\left(B_{K-D}\right)^{I} \longleftrightarrow V=\frac{1}{\sqrt{2}} \psi \in H^{0}(K-D)^{I} \\
1 \in \mathfrak{M}\left(B_{K}\right)^{I} \longleftrightarrow W:=U \otimes V \in H^{0}(K)^{I}
\end{gathered}
$$

and, for $f \in \mathfrak{M}(D)$, let $f U$ denote the holomorphic section in $H^{0}(D)$ corresponding to $f$.

Let $\{e\}$ be a real basis of $H^{0}\left(\mathcal{O}_{X}\right)^{I} \cong \mathbb{R}$. We choose the basis for $H^{0}(D)^{I} \oplus$ $H^{0}(K-D)^{I}$ as follows,

$$
\left\{U, P_{j}^{1} U, P_{j}^{2} U\right\}_{j=1, \ldots, k} \cup\left\{V, Q_{l}^{1} V, Q_{l}^{2} V\right\}_{l=1, \ldots, 2 m-k},
$$

and for $H^{0}(K)^{I} \oplus H^{0}\left(\mathcal{O}_{X}\right)^{I}$,

$$
\left\{W, P_{j}^{1} W, P_{j}^{2} W, Q_{l}^{1} W, Q_{l}^{2} W\right\}_{j=1, \ldots, k}^{l=1, \ldots, 2 m-k} \cup\{e\},
$$


With respect to the above bases, the isomorphism $R_{\mathrm{t}}$ is represented by the matrix

$W$
$W$
$P_{1}^{1} W$
$P_{1}^{2} W$
$\vdots$
$Q_{1}^{1} W$
$Q_{1}^{2} W$
$\vdots$
0 $\left(\begin{array}{cccccccc}U & P_{1}^{1} U & P_{1}^{2} U & \cdots & V & Q_{1}^{1} V & Q_{1}^{2} V & \cdots \\ 0 & 0 & 0 & \cdots & -1 & 0 & 0 & \cdots \\ 0 & -1 & 0 & \cdots & 0 & 0 & 0 & \cdots \\ 0 & 0 & -1 & \cdots & 0 & 0 & 0 & \cdots \\ \vdots & \vdots & \vdots & \ddots & \vdots & \vdots & \vdots & \ddots \\ -\|U\|^{2} & -\left\langle U, P_{1}^{1} U\right\rangle & -\left\langle U, P_{1}^{2} U\right\rangle & \cdots & \|V\|^{2} & \left\langle V, Q_{1}^{1} V\right\rangle & \left\langle V, Q_{1}^{2} V\right\rangle & \cdots\end{array}\right)$

It is easy to see that the determinant of the above matrix is $-\|U\|^{2}-\|V\|^{2}$.

In order to prove any other solution $\mathfrak{t}^{\prime}$ has the same orientation with the solution $\mathfrak{t}$ for $D$ above, we need to prove that the determinant of the matrix $R_{\mathfrak{t}^{\prime}}$ associated with $\mathfrak{t}^{\prime}$ has the same sign with $\operatorname{det} R_{\mathrm{t}}$.

For simplicity, we consider the case when $m=2, k=1$. The generalization to other cases will be obvious. Take the solution $\mathfrak{t}$ corresponding to the divisors $D=\varpi^{*}\left(p_{1}+p_{1}^{*}+p_{2}+p_{2}^{*}\right)$ and $K-D=\varpi^{*}\left(q_{1}+q_{1}^{*}+q_{2}+q_{2}^{*}\right)$. Exchanging $p_{1}$ and $q_{1}$, we obtain another $I$-invariant solution $\mathfrak{t}^{\prime}$ corresponding to $D^{\prime}=\varpi^{*}\left(q_{1}+q_{1}^{*}+p_{2}+p_{2}^{*}\right)$ and $K-D^{\prime}=\varpi^{*}\left(p_{1}+p_{1}^{*}+q_{2}+q_{2}^{*}\right)$. Let $U, V$ be the holomorphic sections for $\mathfrak{t}$, and $U^{\prime}, V^{\prime}$ for $\mathfrak{t}^{\prime}$. Without loss of generality, we may assume $U^{\prime}, V^{\prime}$ are related with $U, V$ by

$$
U^{\prime}=\frac{p_{1}}{q_{1}} \frac{z^{2}-q_{1}^{2}}{z^{2}-p_{1}^{2}} U, \quad V^{\prime}=\frac{q_{1}}{p_{1}} \frac{z^{2}-p_{1}^{2}}{z^{2}-q_{1}^{2}} V .
$$

The meaning of this is as follows: When the holomorphic section $U^{\prime}$ associated with the solution $\mathfrak{t}^{\prime}$ is considered as an element of $H^{0}(D)^{I}=\mathfrak{M}(D)^{I}$ (not of $\left.\mathfrak{M}\left(D^{\prime}\right)^{I}\right)$, it is represented by the meromorphic function $\frac{p_{1}}{q_{1}} \frac{z^{2}-q_{1}^{2}}{z^{2}-p_{1}^{2}}$.

We want to represent the map $R_{\mathfrak{t}^{\prime}}$

$$
R_{\mathrm{t}^{\prime}}\left(\begin{array}{c}
\dot{\phi} \\
\dot{\psi}
\end{array}\right)=\left(\begin{array}{c}
-V^{\prime} \dot{\phi}-U^{\prime} \dot{\psi} \\
\int\left(-\left\langle\dot{\phi}, U^{\prime}\right\rangle+\left\langle\dot{\psi}, V^{\prime}\right\rangle\right) d \operatorname{vol}_{g}
\end{array}\right)
$$

by a matrix with respect to the bases (5.13), (5.14).

Before that we note several useful relations. For $p_{j}$ and $q_{l}$ with $\left|p_{j}\right|=$ $\left|q_{l}\right|=1$, let $a, b$ be the real numbers such that $\frac{p_{j}}{q_{l}}=a+i b$. Then we have the following relations.

$$
\begin{aligned}
P_{j}^{1} Q_{l}^{1} & =-1+\frac{a}{b} P_{j}^{1}-\frac{a}{b} Q_{l}^{1} \\
P_{j}^{1} Q_{l}^{2} & =\frac{1}{b} P_{j}^{2}-\frac{a}{b} Q_{l}^{2} \\
P_{j}^{2} Q_{l}^{1} & =-\frac{1}{b} Q_{l}^{2}+\frac{a}{b} P_{j}^{2} \\
P_{j}^{2} Q_{l}^{2} & =-\frac{1}{b} P_{j}^{1}+\frac{1}{b} Q_{l}^{1}
\end{aligned}
$$

Let $a, b, c, d, e, f$ be the real numbers such that

$$
\frac{p_{1}}{q_{1}}=a+i b, \quad \frac{p_{2}}{q_{1}}=c+i d, \quad \frac{p_{1}}{q_{2}}=e+i f .
$$


Then we have $U^{\prime}=\left(a+b P_{1}^{1}\right) U, V^{\prime}=\left(a-b Q_{1}^{1}\right) V$, and the map $R_{\mathrm{t}^{\prime}}$ is represented by the matrix

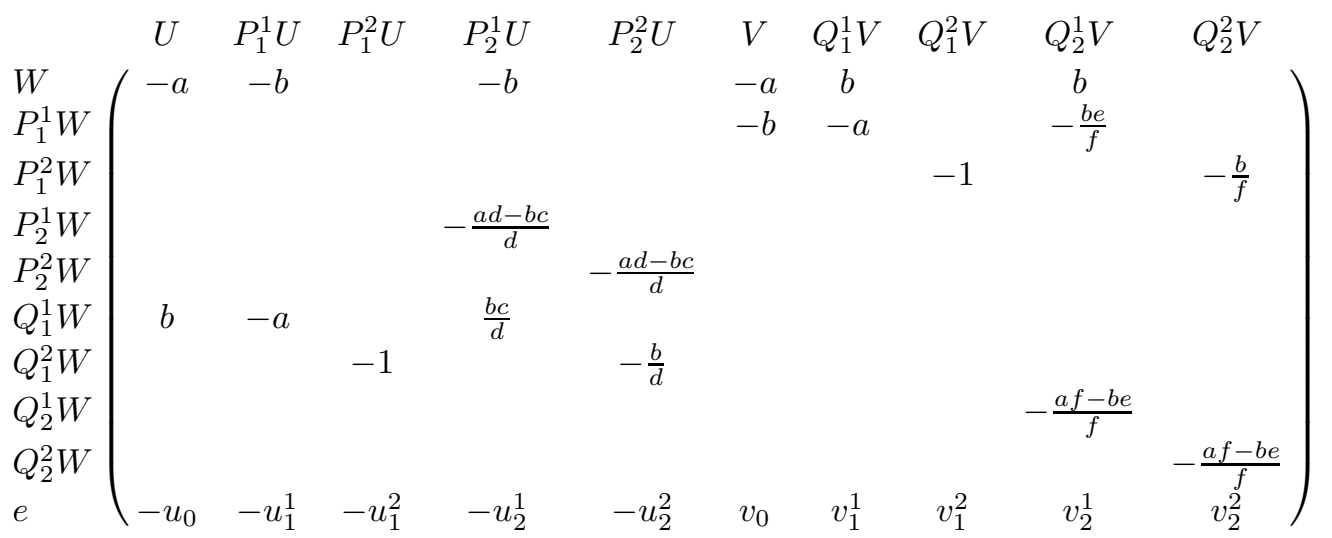

where $u_{0}=\left\langle U, U^{\prime}\right\rangle, u_{j}^{a}=\left\langle P_{j}^{a} U, U^{\prime}\right\rangle, v_{0}=\left\langle V, V^{\prime}\right\rangle, v_{l}^{b}=\left\langle Q_{l}^{b} V, V^{\prime}\right\rangle$. Furthermore, we take one more orientation-preserving basis change given by

$$
\begin{aligned}
& \left(U, P_{1}^{1} U\right)\left(\begin{array}{cc}
a & -b \\
b & a
\end{array}\right)=\left(U^{\prime},\left(-b+a P_{1}^{1}\right) U\right), \\
& \left(V, Q_{1}^{1} V\right)\left(\begin{array}{cc}
a & b \\
-b & a
\end{array}\right)=\left(V^{\prime},\left(b+a Q_{1}^{1}\right) V\right) .
\end{aligned}
$$

Then the matrix above is transformed into

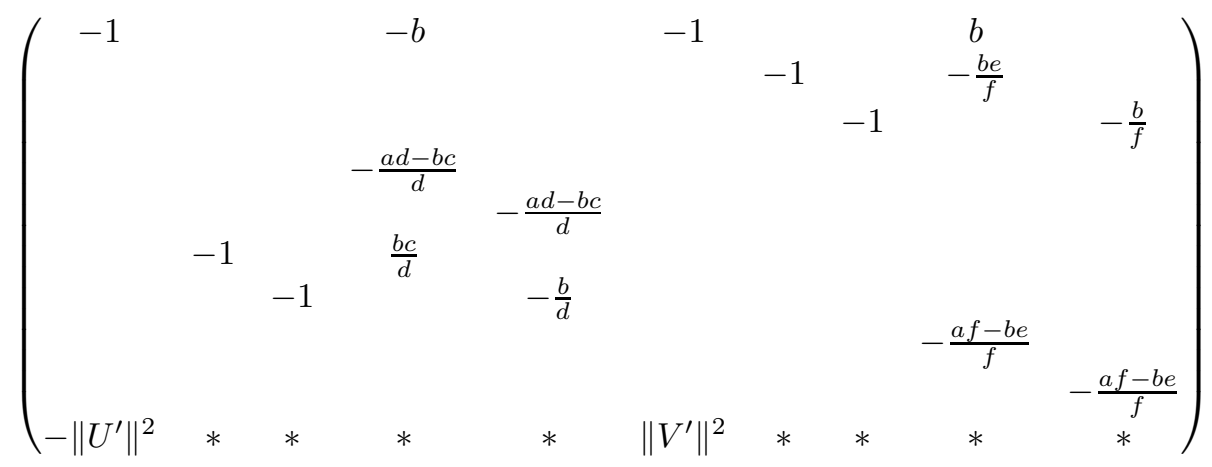

where $*$ are some numbers of inner products of sections. It is easy to see that the determinant of the above matrix is

$$
-\left(\frac{a d-b c}{d}\right)^{2}\left(\frac{a f-b e}{f}\right)^{2}\left(\left\|U^{\prime}\right\|^{2}+\left\|V^{\prime}\right\|^{2}\right) .
$$

Since $\operatorname{det} R_{\mathrm{t}}$ and $\operatorname{det} R_{\mathrm{t}^{\prime}}$ have the same sign, the orientation of the solution $\mathfrak{t}^{\prime}$ is same with that of $\mathfrak{t}$.

The above proof is easily generalized to the cases when $k \neq 1$ or $m \neq 2$, and we see that the orientation does not change by an exchange of some $p_{j}$ and $q_{l}$. Thus Theorem [5.9] is proved.

\section{Concluding remarks}

6.1. More examples with nontrivial $\mathrm{Pin}^{-}(2)$-monopole invariants. By using the gluing formulae in [18, we obtain more examples with nontrivial $\mathrm{Pin}^{-}(2)$-monopole invariants. Let $Z \rightarrow \hat{Z}$ be a nontrivial double covering which satisfies the following: 
(1) $b_{+}^{\ell^{\prime}}(\hat{Z})=0$ for $\ell^{\prime}=Z \times_{\{ \pm 1\}} \mathbb{Z}$.

(2) There is a Spin ${ }^{c_{-}}$structure $\mathfrak{s}^{\prime}$ on $Z \rightarrow \hat{Z}$ whose characteristic bundle $\hat{L}^{\prime}$ satisfies $\tilde{c}_{1}\left(\hat{L}^{\prime}\right)^{2}=\operatorname{sign}(Z)$.

(For instance, a connected sum of several $S^{2} \times \Sigma_{g}$ and $S^{1} \times W$ has a double cover satisfying the above conditions [18, $\S 1.2]$, where $\Sigma_{g}$ is a Riemann surface with genus $g \geq 1$ and $W$ is a closed 3-manifold.) Then [18, Theorem 3.11] implies that $\hat{M}_{4 k} \# \hat{Z}$ and $\hat{X}(4 m+2) \# \hat{Z}$ has nontrivial $\mathrm{Pin}^{-}(2)-$ monopole invariants.

Furthermore, [18, Theorem 3.13] implies that any connected sum $\hat{Y}_{1} \# \cdots \# \hat{Y}_{N}$ such that each $\hat{Y}_{i}$ is $\hat{M}_{4 k}$ or $\hat{X}(4 m+2)$ for any $k$ or $m$ has nontrivial $\mathrm{Pin}^{-}(2)$ monopole invariants.

As an application of the nontriviality of the $\mathrm{Pin}^{-}(2)$-monopole invariants, we have the adjunction inequality for local-coefficient classes [18, Theorem $1.15]$.

6.2. Problems. We suggest several problems for future researches.

- Generalize the results to the case of the real structures with real parts. If we can drop the condition that $\iota$ is free in our story, then we might expect some applications to, say, real algebraic geometry. For this purpose, we need to generalize the notion of the $\mathrm{Spin}^{c_{-}}$-structure.

- Analogy of $\mathrm{SW}=\mathrm{Gr}$ [26]. Can $\mathrm{SW}^{\text {Pin }}$ be identified with some kind of real Gromov-Witten invariant? Cf. Tian-Wang [28].

- What is the counter part of $\mathrm{Pin}^{-}(2)$-monopole equations in Donaldson theory? Is there a version of Witten's conjecture between Pin $^{-}(2)$-monopole theory and Donaldson theory? More concretely, is $\mathrm{SW}^{\mathrm{Pin}}$ equivalent to some kind of Donaldson invariants?

\section{References}

[1] Wolf Barth, Klaus Hulek, Chris Peters, and Antonius Van de Ven, Compact complex surfaces, Ergebnisse der Math. ihrer Grenzgebiete, vol. 4, Springer, 2015.

[2] Olivier Biquard, Les équations de Seiberg-Witten sur une surface complexe non Kählérienne, Communications in Analysis and Geometry 6 (1998), no. 1, 173-197.

[3] Rogier Brussee, The canonical class and the $C^{\infty}$ properties of Kähler surfaces, New York J. Math 2 (1996), 103-146.

[4] Alexander Degtyarev, Ilia Itenberg, and Viatcheslav Kharlamov, Real Enriques surfaces, Lecture Notes in Math., vol. 1746, Springer, 2000.

[5] S. K. Donaldson, Yang-Mills invariants of four-manifolds, Geometry of lowdimensional manifolds, 1 (Durham, 1989), 1990, pp. 5-40.

[6] Robert Friedman and John W Morgan, Algebraic surfaces and Seiberg-Witten invariants, J. Algebraic geom. 6 (1997), 445-479.

[7] _ Obstruction bundles, semiregularity, and Seiberg-Witten invariants, Communications in Analysis and Geometry 7 (1999), no. 3, 451-495.

[8] Akira Fujiki, Kählerian normal complex surfaces, Tohoku Mathematical Journal, Second Series 35 (1983), no. 1, 101-117.

[9] Robert E Gompf and András I Stipsicz, 4-manifolds and Kirby calculus, Graduate Studies in Math., vol. 20, American Mathematical Society Providence, RI, 1999.

[10] Alexandre Grothendieck, Sur quelques points d'algèbre homologique, Tohoku Mathematical Journal, Second Series 9 (1957), no. 2, 119-183.

[11] Nathan Habegger, Une varieté de dimension 4 avec forme d'intersection paire et signature -8, Comm. Math. Helv. 57 (1982), 22-24. 
[12] Ian Hambleton and Matthias Kreck, Smooth structures on algebraic surfaces with cyclic fundamental group, Inventiones mathematicae 91 (1988), no. 1, 53-59.

[13] Jerry L Kazdan and Frank W Warner, Curvature functions for compact 2-manifolds, Annals of Mathematics (1974), 14-47.

[14] Dieter Kotschick, The Seiberg-Witten invariants of symplectic four-manifolds, Séminaire Bourbaki 38 (1995), 195-220.

[15] Dusa McDuff and Dietmar Salamon, Introduction to symplectic topology, Oxford University Press, 1998.

[16] John W Morgan, The Seiberg-Witten Equations and Applications to the Topology of Smooth Four-Manifolds, Mathematical Notes Ser., vol. 44, Princeton University Press, 2014.

[17] Nobuhiro Nakamura, $\mathrm{Pin}^{-}(2)$-monopole equations and intersection forms with local coefficients of four-manifolds, Math. Ann. 357 (2013), no. 3, 915-939.

[18] Pin $^{-}(2)$-monopole invariants, J. Differential Geom. 101 (2015), no. 3, 507549.

[19] Liviu I Nicolaescu, Notes on Seiberg-Witten theory, Graduate Studies in Math., vol. 28, American Mathematical Soc., 2000.

[20] Hiroshi Ohta and Kaoru Ono, Simple singularities and symplectic fillings, Journal of Differential Geometry 69 (2005), no. 1, 001-042.

[21] Robert Silhol, Real algebraic surfaces, Lecture Notes in Math., vol. 1392, Springer, 2006.

[22] Andreas Stieglitz, Equivariant sheaf cohomology, manuscripta mathematica 26 (1978), no. 1-2, 201-221.

[23] Clifford Henry Taubes, The Seiberg-Witten invariants and symplectic forms, Math. Res. Lett. 1 (1994), no. 6, 809-822, DOI 10.4310/MRL.1994.v1.n6.a15. MR1306023 (95j:57039)

[24] More constraints on symplectic forms from Seiberg-Witten invariants, Math. Res. Lett 2 (1995), no. 1, 9-13.

[25] $\quad S W \Rightarrow$ Gr: From the Seiberg-Witten Equations to Pseudo-Holomorphic Curves, Journal of the American Mathematical Society 9 (1996), no. 3, 845-918.

[26] _ Seiberg-Witten and Gromov invariants for symplectic 4-manifolds, International Press Somerville, MA, 2000.

[27] Andrei Teleman, Introduction à la théorie de jauge, Cours spécialisés, SMF, 2012.

[28] Gang Tian and Shuguang Wang, Orientability and real Seiberg-Witten invariants, International Journal of Mathematics 20 (2009), no. 05, 573-604.

[29] Masaaki Ue, Topology of elliptic surfaces, Sugaku 44 (1992).

[30] Shuguang Wang, A vanishing theorem for Seiberg-Witten invariants, Math. Res. Letters 2 (1995), 305-310.

Department of Mathematics, Division of Liberal Arts, Osaka Medical ColLege, 2-7 Daigaku-Machi, Takatsuki City, Osaka, 569-8686, Japan

E-mail address: mat002@osaka-med.ac.jp 